\title{
The computation of Stiefel-Whitney classes
}

\author{
Pierre Guillot
}

November 13, 2018

To the memory of Charles Thomas

\begin{abstract}
L'anneau de cohomologie d'un groupe fini, modulo un nombre premier, peut être calculé à l'aide d'un ordinateur, comme l'a montré Carlson. Ici "calculer" signifie trouver une présentation en termes de générateurs et relations, et seul l'anneau (gradué) sous-jacent est en jeu. Nous proposons une méthode pour déterminer certains éléments de structure supplémentaires: classes de Stiefel-Whitney et opérations de Steenrod. Les calculs sont concrètement menés pour une centaine de groupes (les résultats sont consultables en détails sur Internet).

Nous donnons ensuite une application: à l'aide des nouvelles informations obtenues, nous pouvons dans de nombreux cas déterminer quelles sont les classes de cohomologie qui sont supportées par des cycles algébriques.
\end{abstract}

\begin{abstract}
The cohomology ring of a finite group, with coefficients in a finite field, can be computed by a machine, as Carlson has showed. Here "compute" means to find a presentation in terms of generators and relations, and involves only the underlying (graded) ring. We propose a method to determine some of the extra structure: namely, Stiefel-Whitney classes and Steenrod operations. The calculations are explicitly carried out for about one hundred groups (the results can be consulted on the Internet).

Next, we give an application: thanks to the new information gathered, we can in many cases determine which cohomology classes are supported by algebraic varieties.
\end{abstract}




\section{Contents}

1 Introduction $\quad \mathbf{2}$

1.1 Computer calculations \& Stiefel-Whitney classes . . . . . . . . . 2

1.2 Overview of results . . . . . . . . . . . . . . . . 4

1.3 Strategy \& Organization of the paper . . . . . . . . . 5

2 Formal rings of Stiefel-Whitney classes 7

2.1 Formal rings..................... . . . 7

2.2 Formal properties . . . . . . . . . . . . . . . . . 9

2.3 Chern classes . . . . . . . . . . . . . . . . . . . . . . . . . . . . . . . . . . . 13

2.4 Steenrod operations ... . . . . . . . . . . . . . 13

2.5 The $\operatorname{ring} \mathcal{W}_{F}^{*}(G) \ldots \ldots \ldots \ldots$

3 The main algorithm $\quad 15$

3.1 Notations \& Preliminaries . . . . . . . . . . . . . . . . . . . . . . 15

3.2 Construction of certain maps $\mathcal{W}_{F}^{*}(G) \rightarrow H^{*}(G) \ldots$. . . . . . . 17

3.3 Tests \& Conclusions . . . . . . . . . . . . . . . . . . . . 19

3.4 Comments....................... 21

4 Experimental results $\quad 22$

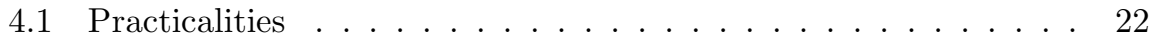

4.2 Mathematical results .................. 23

5 Application to algebraic cycles $\quad \mathbf{2 4}$

5.1 Algebraic cycles in the cohomology . . . . . . . . . . . . 24

5.2 Computations . . . . . . . . . . . . . . . . 25

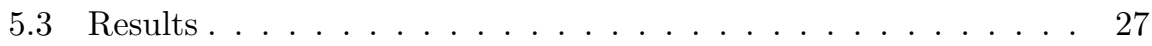

A Theoretical considerations $\quad 29$

A.1 The Atiyah-Evens approach . . . . . . . . . . . . . . . . . 29

A.2 The Thom construction . . . . . . . . . . . . . . . 30

A.3 The finite field trick . . . . . . . . . . . . . . . . 32

\section{§1. Introduction}

\subsection{Computer calculations \& Stiefel-Whitney classes}

For a long time, it was very common for papers on group cohomology to point out the lack of concrete, computational examples in the subject (see for example the introduction to [25]). Since then, the situation has dramatically changed with the observation by Carlson (see [7]) that the cohomology ring could be computed in finite time, by an algorithmic method for which a computer could be trusted. The reader can check on the Internet (see [2] and [3]) the myriad of examples of cohomology rings which have now been obtained.

The question arises then: can we exploit those calculations to tackle some problems related to the cohomology of groups? The particular problem which originally motivated me (and which, as it turned out, was to play only a secondary role in this paper) was the following. Since Totaro's paper [26], it is known that the classifying space $B G$ of a finite group $G$ is a limit of algebraic 
varieties (say, over $\mathbb{C}$ ), and thus one can ask for a description of the image of the map

$$
C H^{*} B G \rightarrow H^{*}(B G, \mathbb{Z})
$$

where $C H^{*} B G$ is the Chow ring of $B G$. It is similar to the question posed by the Hodge conjecture, but with some distinctive features (for example $C H^{*} B G$ is all torsion when $G$ is finite, so we cannot be content with a description of the map above after tensoring with $\mathbb{Q}$ ).

However, a description of $H^{*}\left(B G, \mathbb{F}_{p}\right)$ as a ring, which is what the computer provides, if of little help vis-à-vis this problem, and many others. In any case, let us compare the sort of output produced by the computer with a more traditional answer.

Let us focus on the example of $Q_{8}$, the quaternion group of order 8. At the address [2], one will find that $H^{*}\left(B Q_{8}, \mathbb{F}_{2}\right)$ is an algebra on generators $z, y, x$ of degree $1,1,4$ respectively, subject to the relations $z^{2}+y^{2}+z y=0$ and $z^{3}=0$. One also finds a wealth of information on subgroups of $Q_{8}$ and their cohomology, calculations of transfers and restrictions, as well as a thorough treatment of the commutative algebra of $H^{*}\left(B Q_{8}, \mathbb{F}_{2}\right)$ (nilradical, Krull dimension, etc).

On the other hand, if we look at the computation by Quillen of the cohomology of extraspecial groups (see [22]), one finds in the case of $Q_{8}$ :

Proposition 1.1 - There are 1-dimensional, real representations $r_{1}$ and $r_{2}$ of $Q_{8}$, and a 4-dimensional representation $\Delta$, such that $H^{*}\left(B Q_{8}, \mathbb{F}_{2}\right)$ is generated by $w_{1}\left(r_{1}\right), w_{1}\left(r_{2}\right)$ and $w_{4}(\Delta)$. The ideal of relations is generated by $R=w_{1}\left(r_{1}\right)^{2}+w_{1}\left(r_{2}\right)^{2}+w_{1}\left(r_{1}\right) w_{1}\left(r_{2}\right)$ and $S q^{1}(R)$.

Finally, $S q^{1}(\Delta)=S q^{2}(\Delta)=S q^{3}(\Delta)=0$.

This calls for several comments. First, if $r$ is any real representation of a (finite or compact Lie) group $G$, then $r$ can be seen as a homomorphism $r: G \rightarrow O(n)$ where $n$ is the real dimension of $r$. This yields a continuous map $B r: B G \rightarrow B O(n)$ and thus a ring homomorphism $B r^{*}: H^{*}\left(B O(n), \mathbb{F}_{2}\right) \rightarrow$ $H^{*}\left(B G, \mathbb{F}_{2}\right)$. The ring $H^{*}\left(B O(n), \mathbb{F}_{2}\right)$ is polynomial on variables $w_{1}, \ldots, w_{n}$, and the element $\mathrm{Br}^{*}\left(w_{i}\right)$ is written $w_{i}(r)$ and called the $i$-th Stiefel-Whitney class of $r$, a central object of study in this paper (more details on the definition follow).

Second, the cohomology ring of any space in an unstable algebra, and is acted on by the Steenrod operations $S q^{k}, k \geq 0$. This gives much structure on the cohomology, as will be examplified below. For the time being, we point out that the presentation of the cohomology of $Q_{8}$ is simplified by the use of Steenrod operations, in the sense that $R$ is the only significant relation, the other one being obtained by applying $S q^{1}$.

Note that these two things are related, for one knows how to compute the Steenrod operations on $H^{*}\left(B O(n), \mathbb{F}_{2}\right)$ via $W u$ 's formula, see [20]. Since $B r^{*}$ commutes with the $S q^{k}$, one knows how these operations act on the StiefelWhitney classes. Once we know that the cohomology of a group is generated by such classes, as is the case for $Q_{8}$, we get all the information on Steenrod operations for free.

Note also, finally, that Stiefel-Whitney classes give some geometric or representation - theoretic meaning to the relations in the cohomology of a group, in good cases. In the case of $Q_{8}$ thus, there is a relation between the representa- 
tions mentioned in proposition 1.1, namely:

$$
\lambda^{2}(\Delta)=r_{1}+r_{2}+r_{1} \otimes r_{2}+3
$$

(here " +3 " means three copies of the trivial representation, and $\lambda^{2}$ means the second exterior power). There are formulae expressing the Stiefel-Whitney classes of a direct sum, a tensor product, or an exterior power: these will be recalled in section 2 . In the present case, they give $w_{2}\left(r_{1}+r_{2}+r_{1} \otimes r_{2}+3\right)=$ $w_{1}\left(r_{1}\right)^{2}+w_{1}\left(r_{2}\right)^{2}+w_{1}\left(r_{1}\right) w_{1}\left(r_{2}\right)$, while $w_{2}\left(\lambda^{2}(\Delta)\right)=0$. The latter takes into account the fact that $w_{1}(\Delta)=w_{2}(\Delta)=w_{3}(\Delta)=0$, which in turn is a formal consequence of the fact that $\Delta$ carries a structure of $\mathbb{H}$-module, where $\mathbb{H}$ is the algebra of quaternions. Putting all this together, we get an "explanation" for the relation $w_{1}\left(r_{1}\right)^{2}+w_{1}\left(r_{2}\right)^{2}+w_{1}\left(r_{1}\right) w_{1}\left(r_{2}\right)=0$ based on representation theory.

All this extra decoration on the cohomology ring is extremely useful. For example if we return to the problem, already alluded to, of computing which cohomology classes are supported by algebraic varieties, then we have a lot to learn from this new information. The Chern classes, which are analogous to Stiefel-Whitney classes but related to complex representations rather than real ones, and which can be computed mod 2 from the Stiefel-Whitney classes, are always supported by algebraic varieties; this gives a "lower bound". On the other hand, classes coming from the Chow ring are killed by certain Steenrod operations, and this gives an "upper bound". See $\S 5$ for details.

The main purpose of this paper is to describe a method for the systematic computation of Stiefel-Whitney classes, mostly with the help of a computer. Let us describe our success in the matter.

\subsection{Overview of results}

This paper has a companion, in the form of a computer program. The source and the results of the computer runs can be consulted at [1]. We encourage the reader to have a look at this page now. The present paper can largely be seen as an explanation of the program, although it can by all means be read independently.

It is in the nature of our algorithm that it does not work in all cases. On the brighter side, it is very much simpler than any full-blown method for calculating Stiefel-Whitney classes in general (see the Appendix for a discussion of possible approaches to the general problem). Also, our basic method can be adjusted for specific groups and made to work in new cases by small, taylored improvements. Our original goal however was to constitute, if not a "database", at least a significant collection of examples (rather than deal with a handful of important groups).

We have focused on the groups of order dividing 64 . We got a full answer for the 5 groups of order 8 , for 13 of the 14 groups of order 16 , for 28 of the 51 groups of order 32 , and for 61 of the 267 groups of order 64 . Thus we were able to deal with more than 100 groups.

Obtaining a "full answer" means the following. When $H^{*}\left(B G, \mathbb{F}_{2}\right)$ is generated by Stiefel-Whitney classes, the computer proves it, and gives the same sort of information as in proposition 1.1. When $H^{*}\left(B G, \mathbb{F}_{2}\right)$ is not generated by Stiefel-Whitney classes, the answer looks as follows. Let us the consider the smallest example, which is that of the group of order 16 whose Hall-Senior 
number is 11 . This group is the semidirect product $\mathbb{Z} / 8 \rtimes \mathbb{Z} / 2$ whose centre is a $\mathbb{Z} / 4$. The computer output is:

Proposition 1.2 - The cohomology of $G$ is generated by $w_{1}\left(r_{2}\right), w_{1}\left(r_{3}\right), w_{4}\left(r_{8}\right)$ and a class $x$ of degree 3 which is not in the subring generated by Stiefel-Whitney classes. Here $r_{2}$ and $r_{3}$ are 1-dimensional real representations, while $r_{8}$ is a 4dimensional representation of complex type. The relations are

$$
\begin{gathered}
w_{1}\left(r_{2}\right)^{2}+w_{1}\left(r_{3}\right)^{2}=0, \quad w_{1}\left(r_{2}\right)^{2} w_{1}\left(r_{3}\right)+w_{1}\left(r_{2}\right) w_{1}\left(r_{3}\right)^{2}=0, \\
w_{1}\left(r_{2}\right) x+w_{1}\left(r_{3}\right) x=0, \quad x^{2}=0 .
\end{gathered}
$$

Moreover one has $S q^{1}\left(w_{4}\left(r_{8}\right)\right)=0, S q^{2}\left(w_{4}\left(r_{8}\right)\right)=w_{4}\left(r_{8}\right) w_{1}\left(r_{2}\right) w_{1}\left(r_{3}\right)$, and $S q^{3}\left(w_{4}\left(r_{8}\right)\right)=0$.

Finally, the element $x$ is the same as the $x$ in Carlson's presentation for $H^{*}(G)$.

The only piece of information missing is the action of the Steenrod operations on $x$. However, one can recover this "by hand", knowing that $x$ is the same as Carlon's $x$ : indeed, on Carlson's page [2] we see that $x$ is a transfer of an (explicitly given) element in the cohomology of an elementary abelian 2subgroup of $G$. Transfers commute with Steenrod operations, and we deduce easily the value of $S q^{1} x$ and $S q^{2} x$.

The computer also provides some other details, for example all the Chern classes, and all the other Stiefel-Whitney classes, are given in this presentation for $H^{*}(G)$.

Turning to the application to algebraic cycles, there are 38 groups for which we describe the image of $C H^{*} B G \rightarrow H^{*}(G)$. For example when $G=Q_{8}$ this image is generated by $w_{1}\left(r_{1}\right)^{2}, w_{1}\left(r_{2}\right)^{2}$ and $w_{4}(\Delta)$. There are 62 groups in total for which we provide at least partial information on algebraic cycles, see $\S 5$.

\subsection{Strategy \& Organization of the paper}

Given a group $G$, we shall always assume that we have a presentation of $H^{*}\left(B G, \mathbb{F}_{2}\right)$ as a ring available (as proposition 1.2 suggests, we have chosen to get this information from Carlson's webpage). We shall then define a ring $\mathcal{W}_{F}^{*}(G)$ as follows. As a graded $\mathbb{F}_{2}$-algebra, $\mathcal{W}_{F}^{*}(G)$ is to be generated by formal variables $w_{j}\left(r_{i}\right)$ where the $r_{i}$ 's are the irreducible, real representations of $G$. Then we impose all the relations between these generators which the theory of Stiefel-Whitney classes predicts: relations coming from the formulae for tensor products and exterior powers, rationality conditions, and so on. (It is perhaps more accurate to say that we impose all the relations that we can think of.)

Then one has a map $a: \mathcal{W}_{F}^{*}(G) \rightarrow H^{*}\left(B G, \mathbb{F}_{2}\right)$ with good properties: namely, it is an isomorphism in degree 1 , and turns the cohomology of $G$ into a finitely generated module over $\mathcal{W}_{F}^{*}(G)$. The key point is that, in fact, there are very few maps between these two rings having such properties (in practice, there are so many relations in $\mathcal{W}_{F}^{*}(G)$ that there are few well-defined maps out of this ring anyway).

The slight twist here is that, unlike what you might expect, we do not compute the effect of the map a. Rather, we write down an exhaustive list of all the maps $\mathcal{W}_{F}^{*}(G) \rightarrow H^{*}\left(B G, \mathbb{F}_{2}\right)$ having the same properties as $a$, and it turns out, most of the time, that all these maps have the same kernel and "essentially" 
the same image (the word "essentially" will be justified later). More often than not, all the maps are surjective; let us assume in this introduction that it is so for a given $G$, postponing the more difficult cases. Since $a$ is among these maps (without our knowing which one it is!), we know its kernel, and we have a presentation of $H^{*}\left(B G, \mathbb{F}_{2}\right)$ as a quotient of $\mathcal{W}_{F}^{*}(G)$, that is a presentation in terms of Stiefel-Whitney classes. The computation of Steenrod operations becomes trivial.

As a toy example, we may come back to $G=Q_{8}$. In this case one has

$$
\mathcal{W}_{F}^{*}(G)=\frac{\mathbb{F}_{2}\left[w_{1}\left(r_{1}\right), w_{1}\left(r_{2}\right), w_{4}(\Delta)\right]}{\left(R, S q^{1}(R)\right)}
$$

where $R=w_{1}\left(r_{1}\right)^{2}+w_{1}\left(r_{2}\right)^{2}+w_{1}\left(r_{1}\right) w_{2}\left(r_{2}\right)$. It is apparent that $\mathcal{W}_{F}^{*}(G)$ is abstractly isomorphic with $H^{*}\left(G, \mathbb{F}_{2}\right)$; Quillen's theorem states much more specifically that the map $a$ is an isomorphism. Our approach, reducing to something trivial here, is to note that there are only two classes in degree 4 in the cohomology ring, namely 0 and an element $x$ which generates a polynomial ring. If the image under $a$ of the Stiefel-Whitney class $w_{4}(\Delta)$ were 0 , then $H^{*}\left(G, \mathbb{F}_{2}\right)$ could not be of finite type over $\mathcal{W}_{F}^{*}(G)$. Thus $a\left(w_{4}(\Delta)\right)=x$. Since $a$ is an isomorphism in degree 1, it must be surjective; for reasons of dimensions it is an isomorphism. In this fashion we recover Quillen's result from the presentation of the cohomology as given by Carlson and a simple game with $\mathcal{W}_{F}^{*}(G)$, and this (in spirit if not in details) is what our program will do. Now, describing $\mathcal{W}_{F}^{*}(G)$ explicitly is extremely long if one proceeds manually, but it is straightforward enough that a computer can replace us.

We insist that we are not able to give an expression for the Stiefel-Whitney classes in terms of the generators originally given in the presentation of $H^{*}\left(B G, \mathbb{F}_{2}\right)$ that we start with. Thus we do not "compute" the Stiefel-Whitney classes in the sense that one might have expected. For this reason, we have found it worthwile to collect in an Appendix a review of the methods that one could use in order to actually perform these computations (in the sense, say, of obtaining cocycle representatives for the Stiefel-Whitney classes relative to a given projective resolution). Our objective is twofold: on the one hand, we hope to convince the reader that these computations are considerably difficult indeed, and that we should be so lucky to have a "trick" to avoid them; on the other hand, we also hope that the suggestions we make in the Appendix will actually be useful to anyone wishing to take up the challenge. We describe three ways to attack the calculations, none of which I have seen presented in the literature as a computational device (though they each rely on classical results).

It is perhaps useful at this point to comment on the logic underlying this paper. After reflecting on the difficulties arising in the computation of StiefelWhitney classes, as exposed in the Appendix, one wishes to calculate a minimal number of them. Certainly if a representation can be expressed in terms of others using direct sums, tensor products and exterior powers, then there is no need to compute its Stiefel-Whitney classes separately. The $\operatorname{ring} \mathcal{W}_{F}^{*}(G)$ was originally designed to keep track of all such redundancies in a compact way. Subsequently, it has come as a genuine surprise that this ring made the computations so much simpler that, in many cases, there was nothing left to do. 
$\diamond$ The paper is organized as follows. In section 2, we introduce the ring $\mathcal{W}_{F}^{*}(G)$, whose definition is a bit lengthy. The map a will appear naturally. In section 3, we describe in details our algorithm to find a presentation for the cohomology of $G$ with the help of $\mathcal{W}_{F}^{*}(G)$, as outlined above. In section 4 , we comment on the experimental results which we have had. Finally in section 5, we apply the preceding results to the study of the "cycle map" between the Chow ring and the cohomology of $B G$.

$\diamond$ Acknowledgements. It is a pleasure to thank David Green and Jon Carlson for their early interest in this work. I am also grateful to Alain Sartout and Pierre Navarro for their patience and help with the servers here in Strasbourg. My thanks extend to William Stein for advertising this work on the SAGE website, and for being generally helpful on the SAGE forum.

This project would have been impossible to realize without the help of many wonderful software packages. Crucial use was made of the GAP algebra system, the Python programming language, and the SAGE mathematical suite. Many tools coming from the GNU Free Software Foundation have been essential, most of all the $\mathrm{g}++$ compiler. (I could also mention GNU/linux in general, emacs, and also Latex.)

No use was made of any commercial software.

$\diamond$ This paper is dedicated to the memory of Charles Thomas. Charles lectured me on Lie groups when I was a graduate student, and was always available for challenging discussions during my PhD. He was very fond of characteristic classes, and explained on many occasions how the $\lambda$-ring structure on the representation ring of a group was a much under-used tool. I hope that the computations which follow, for which the $\lambda$-operations play such a key role, would have had some appeal to him.

\section{$\S 2$. Formal rings of Stiefel-Whitney classes}

From now on, we shall write $H^{*}(G)$ for the mod 2 cohomology of the finite group $G$. Occasionally we may use the notation $H^{*}(B G)$ in order to emphasize a topological context.

\subsection{Formal rings}

Let $r_{1}, \ldots, r_{m}$ denote the isomorphism classes of real, irreducible representations of $G$, and let $n_{i}$ be the real dimension of $r_{i}$. Each $r_{i}$ gives rise, by choice of a basis and a $G$-invariant inner product, to a homomorphism $G \rightarrow O\left(n_{i}\right)=$ $O_{n_{i}}(\mathbb{R})$. The latter is well-defined up to conjugacy in $O\left(n_{i}\right)$, and we also use the notation $r_{i}$ for any choice of homomorphism. Note that the homotopy class of $B r_{i}: B G \rightarrow B O\left(n_{i}\right)$ is also well-defined.

Consider now the ring

$$
A_{G}^{*}=\bigotimes_{i=1}^{m} H^{*}\left(B O\left(n_{i}\right)\right) .
$$


This $A_{G}^{*}$ is a polynomial ring on generators which we write $\bar{w}_{j}\left(r_{i}\right)$, for $1 \leq i \leq m$ and $1 \leq j \leq n_{i}$. There is a natural map

$$
\pi=\pi_{G}: A_{G}^{*} \rightarrow H^{*}(B G)
$$

obtained by tensoring together the induced maps $B r_{i}^{*}$. The image of $\bar{w}_{j}\left(r_{i}\right)$ under $\pi$ is of course $w_{j}\left(r_{i}\right)$, the $j$-th Stiefel-Whitney class of $r_{i}$.

There is a modest interpretation of $A_{G}^{*}$ (and $\pi$ ) in "universal" terms. For this, we need some notations. In the presence of a graded $\operatorname{ring} R^{*}$, we write $R^{\times}$ for the group of elements $\left(a_{n}\right)$ in the product $\prod_{n} R^{n}$ such that $a_{0}=1$. We write such elements $1+a_{1}+a_{2}+\cdots$ and multiply them in the obvious way. Then, writing $R_{\mathbb{R}}(G)$ for the real representation ring of $G$, the total Stiefel-Whitney class is the group homomorphism

$$
\begin{aligned}
w: \quad R_{\mathbb{R}}(G) & \longrightarrow H^{\times}(G), \\
\rho & \mapsto 1+w_{1}(\rho)+w_{2}(\rho)+\cdots
\end{aligned}
$$

defined by sending the generator $r_{i}$ of the free abelian group $R_{\mathbb{R}}(G)$ to $1+$ $w_{1}\left(r_{i}\right)+w_{2}\left(r_{i}\right)+\cdots$. This extends the above definition of $w_{j}(-)$ to representations which are not necessarily irreducible (and even to virtual representations). Of course we could also have given an extended definition directly for an arbitrary representation, exactly as above: it is then a nontrivial, but very well-known, fact that the two definitions coincide.

Consider now the following diagram:

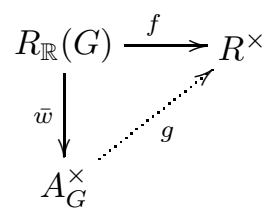

Here $R^{*}$ is any graded ring, and $R^{\times}$is as above, while $f$ is any group homomorphism such that $f\left(r_{i}\right)$ is zero in degrees greater than $n_{i}$. The map $\bar{w}$ sends $r_{i}$ to $1+\bar{w}_{1}\left(r_{i}\right)+\bar{w}_{2}\left(r_{i}\right)+\cdots$. The universality of $\bar{w}$ can be expressed by saying that the dotted arrow $g$ always exists, making the triangle commute. What is more, $g$ always comes from an underlying map of graded rings $A_{G}^{*} \rightarrow R^{*}$, and the latter is unique.

Taking $R^{*}=H^{*}(G)$ and $f=w$, the map $\pi$ can then be seen as being induced by universality.

This brings us to the following definition. Any ring which is obtained as a quotient of $A_{G}^{*}$ by an ideal contained in ker $\pi$ will be called a formal ring of Stiefel-Whitney classes. As the name suggests, we shall obtain examples of such rings by looking at formal properties of Stiefel-Whitney classes, as we have just done with the property " $w_{j}\left(r_{i}\right)=0$ when $j>n_{i}$ ". Each example $F^{*}$ will come equipped with a map $R_{\mathbb{R}}(G) \rightarrow F^{\times}$which is universal among certain maps, but we shall leave to the reader this interpretation.

An extreme example of formal ring, thus, is $A_{G}^{*} / \operatorname{ker} \pi$, which we denote by $\mathcal{W}^{*}(G)$. It can be thought of as a subring of $H^{*}(G)$, namely the subring generated by all the Stiefel-Whitney classes. Eventually we shall end up being able to compute $\mathcal{W}^{*}(G)$ in many cases, and our main tool is the use of other formal rings, which we use as approximations to $\mathcal{W}^{*}(G)$. 


\subsection{Formal properties}

The definition of $A_{G}^{*}$ (in universal terms) uses only the fact that $w_{j}\left(r_{i}\right)$ vanishes when $j$ is large, and implicitly the formula for the Stiefel-Whitney classes of a direct sum (in that $w$ is a group homomorphism). We shall now review the other familiar properties of Stiefel-Whitney classes.

$\diamond$ Rationality. Let $V$ be a real and irreducible representation of $G$. Schur's lemma says that $K=\operatorname{End}_{G}(V)$ is a field (not necessarily commutative). Since $K$ must contain $\mathbb{R}$ in its centre, it follows that $K$ must be one of $\mathbb{R}, \mathbb{C}$ or $\mathbb{H}$. Accordingly, $V$ is said to be of real, complex, or quaternion type.

The consequences on Stiefel-Whitney classes are as follows. If $r_{i}$ is of complex type, then $r_{i}: G \rightarrow O\left(n_{i}\right)$ can be factorized as a composition

$$
G \rightarrow U\left(d_{i}\right) \rightarrow O\left(n_{i}\right)
$$

where $U\left(d_{i}\right)$ is the unitary group, $n_{i}=2 d_{i}$, and the second arrow is realification. Thus we can also write:

$$
B r_{i}^{*}: H^{*} B O\left(n_{i}\right) \rightarrow H^{*} B U\left(d_{i}\right) \rightarrow H^{*} B G .
$$

Since the cohomology of $B U\left(d_{i}\right)$ is concentrated in even degrees, we conclude that $w_{2 j+1}\left(r_{i}\right)=0$ when $r_{i}$ is of complex type.

Similarly, when $r_{i}$ is of quaternion type, we have $w_{j}\left(r_{i}\right)=0$ whenever $j$ is not divisible by 4 , for the cohomology of $B S p\left(d_{i}\right)$ is concentrated in degrees divisible by 4 . Here $S p\left(d_{i}\right)$ is the symplectic group and $n_{i}=4 d_{i}$.

It is very easy to check whether a given representation is of complex or quaternion type, see [24], §13.2. In this way we obtain with little effort a collection of elements of the form $\bar{w}_{j}\left(r_{i}\right)$ in $A_{G}^{*}$ which all belong to ker $\pi$.

$\diamond$ Before we proceed, we need to recall the splitting principle. This says roughly that everything happens as if any representation were a direct sum of 1-dimensional representations, as far as computing the Stiefel-Whitney classes goes. More precisely, given real representations $\alpha$ and $\beta$ of dimensions $n_{\alpha}$ and $n_{\beta}$ respectively, one may find an injection of $H^{*}(G)$ into a ring in which we have factorizations

$$
w(\alpha)=\prod_{k=1}^{n_{\alpha}}\left(1+a_{k}\right)
$$

and

$$
w(\beta)=\prod_{\ell=1}^{n_{\beta}}\left(1+b_{\ell}\right)
$$

where each $a_{k}$ and $b_{\ell}$ has degree 1 . Thus one recovers $w_{n}(\alpha)$ as the $n$-th elementary symmetric function in the "roots" $a_{k}$, and likewise for $\beta$. The formulae below will be given in terms of the roots. This traditional choice avoids introducing lots of universal polynomials with awkward names. 
$\diamond$ Tensor products. One has the following well-known formula:

$$
w(\alpha \otimes \beta)=\prod_{\substack{1 \leq k \leq n_{\alpha} \\ 1 \leq \ell \leq n_{\beta}}}\left(1+a_{k}+b_{\ell}\right)
$$

The reader should notice that the formula is strictly associative, in the sense that the two universal formulae for the total Stiefel-Whitney class of $\alpha \otimes \beta \otimes \gamma$ which you could deduce from the result above would be precisely the same. Likewise, it is strictly commutative. The fact that the tensor product operation is associative and commutative up to isomorphism only guarantees, a priori, that the formula is associative and commutative in $H^{*}(G)$, for all $G$; since we can consider the universal example of orthogonal groups and their defining representations, however, this is enough. We shall use trivial remarks of this sort without comments in the sequel. They are of some importance nonetheless, as we sometimes work in the ring $A_{G}^{*}$ before applying $\pi$ to reach $H^{*}(G)$.

To exploit this, we look at the presentation

$$
R_{\mathbb{R}}(G)=\mathbb{Z}\left[r_{1}, \ldots, r_{m}\right] / \mathfrak{a} .
$$

For any $x \in \mathfrak{a}$, we wish to obtain a relation $T(x) \in A_{G}^{*}$ which lies in $\operatorname{ker} \pi$. We need some care to make sure that the computation can be done in finite time, and in particular we want to avoid the computation of inverses of elements in the group $A_{G}^{\times}$. We proceed thus: write $x=P-Q$ where $P$ is the sum of the terms of $x$ which have positive coefficients. Then $Q$ also has positive coefficients. One may obtain an element $T_{P}$ in $A_{G}^{\times}$by computing the total Stiefel-Whitney class of each term of $P$ according to the rule above for tensor products, and then multiply out (in $A_{G}^{\times}$) the results for the various terms. Proceed similarly for $T_{Q}$, working with $Q$ instead of $P$. Then put $T(x)=T_{P}-T_{Q}$ (which is also $T_{Q}-T_{P}$ as we are in characteristic 2). That $T(x) \in \operatorname{ker} \pi$ follows from the fact that $x=0$ in $R_{\mathbb{R}}(G)$ and the fact that the formula for tensor products indeed holds, in $H^{*}(G)$.

We note that, if one writes $x=P^{\prime}-Q^{\prime}$ for any $P^{\prime}$ and $Q^{\prime}$ having positive coefficients, then $P^{\prime}=P+S$ and $Q^{\prime}=Q+S$ for some polynomial $S$. Then $T_{P^{\prime}}-T_{Q^{\prime}}=T_{S}\left(T_{P}-T_{Q}\right)$. Since $T_{S}$ is a unit in every truncated ring $A_{G}^{<N}$, it follows that $T(x)=u\left(T_{P^{\prime}}-T_{Q^{\prime}}\right)$ (here $u$ is a truncation of $T_{S}^{-1}$ ). We use this in the proof of lemma 2.2 below.

ExAmple 2.1 - Let $G=\mathbb{Z} / 4$. Then $G$ has three real, irreducible representations: the trivial one, the one-dimensional representation $\alpha$ coming from the projection $\mathbb{Z} / 4 \rightarrow \mathbb{Z} / 2$, and the 2 -dimensional representation $\beta$ obtained by viewing $G$ as the group of 4 -th roots of unity in $\mathbb{C}$.

We have

$$
R_{\mathbb{R}}(G)=\frac{\mathbb{Z}[\alpha, \beta]}{\left(\alpha^{2}-1, \beta^{2}-2 \alpha-2, \alpha \beta-\beta\right)} .
$$

Consider the relation $\alpha \beta=\beta$. The formula for tensor products gives in this case $w(\alpha \beta)=1+w_{1}(\beta)+w_{1}(\alpha) w_{1}(\beta)+w_{2}(\beta)$. This being equal to the total Stiefel-Whitney class of $\beta$, we have therefore $w_{1}(\alpha) w_{1}(\beta)=0$. In other words

$$
T(\alpha \beta-\beta)=\bar{w}_{1}(\alpha) \bar{w}_{1}(\beta) \in \operatorname{ker} \pi .
$$


Similarly, looking at $\beta^{2}=2 \alpha+2$ gives the relation $w_{1}(\alpha)^{2}=w_{1}(\beta)^{2}$, and the element $T\left(\beta^{2}-2 \alpha-2\right)=\bar{w}_{1}(\alpha)^{2}-\bar{w}_{1}(\beta)^{2}$ is in $\operatorname{ker} \pi$. The relation $\alpha^{2}=1$ gives nothing.

Now, the representation $\beta$ has a complex structure, of course. It follows that $w_{1}(\beta)=0$, and we will find the element $\bar{w}_{1}(\beta)$ in $\operatorname{ker} \pi$.

Combining all this, we see that $\operatorname{ker} \pi$ contains $\bar{w}_{1}(\beta)$ and $\bar{w}_{1}(\alpha)^{2}$. Of course the cohomology of $\mathbb{Z} / 4$ is known, and it turns out that ker $\pi$ is precisely generated by these two elements. So in this simple case all of $\operatorname{ker} \pi$, and indeed all the relations in the cohomology, are explained by representation theory.

All the information available can be got in finite time:

LEMma 2.2 - If $x_{1}, \ldots, x_{n}$ generate $\mathfrak{a}$, then any element of the form $T(x)$ for $x \in \mathfrak{a}$ is in the ideal generated by the homogeneous parts of the elements $T\left(x_{1}\right), \ldots, T\left(x_{n}\right)$ in $A_{G}^{*}$.

Proof. If $x$ and $y$ are in $\mathfrak{a}$, then let $x=P-Q$ and $y=P^{\prime}-Q^{\prime}$ as above. We have $T(x+y)=u\left(T_{P+P^{\prime}}-T_{Q+Q^{\prime}}\right)$. However $T_{P+P^{\prime}}=T_{P} T_{P^{\prime}}$, a product in $A_{G}^{\times}$ or rather a product of non-homogeneous elements in $A_{G}^{*}$; similarly for $Q$. Thus

$$
T(x+y)=u T_{P}\left(T_{P^{\prime}}-T_{Q^{\prime}}\right)+u T_{Q^{\prime}}\left(T_{P}-T_{Q}\right)=u T_{P} T(y)+u T_{Q^{\prime}} T(x) .
$$

So $T(x+y)$ is in the ideal generated by $T(x)$ and $T(y)$.

Further, $T(x)=T(-x)$ clearly.

Finally, assume $x$ is in $\mathfrak{a}$ and $y=r_{k}$ for some $k$. Write $x=P-Q$. Then $T(x y)=T_{P y}-T_{Q y}$. From the above we see that there is a universal polynomial $f$ such that $T_{P y}=f\left(T_{P}^{(1)}, T_{P}^{(2)}, \ldots, w_{1}(y), w_{2}(y), \ldots\right)$, where $T_{P}^{(i)}$ is the degree $i$ homogeneous part of $T_{P}$; moreover the same $f$ has also $T_{Q y}=f\left(T_{Q}^{(1)}, T_{Q}^{(2)}, \ldots, w_{1}(y), w_{2}(y), \ldots\right)$. It is then clear that $T(x y)$ is in the ideal generated by the various $T_{P}^{(i)}-T_{Q}^{(i)}$, which are the homogeneous parts of $T(x)$.

This completes the proof.

$\diamond$ Exterior powers. We recall the following.

$$
w\left(\lambda^{p} r_{i}\right)=\prod_{1 \leq i_{1}<\cdots<i_{p} \leq n_{i}}\left(1+a_{i_{1}}+\cdots+a_{i_{p}}\right) .
$$

So the structure of $\lambda$-ring on $R_{\mathbb{R}}(G)$ will give us relations between the StiefelWhitney classes. Now, the whole $\lambda$-ring structure is entirely described by the value of $\lambda^{p}\left(r_{i}\right)$ for $1 \leq p \leq n_{i}$, for there are universal polynomials expressing $\lambda^{p}(x+y)$ and $\lambda^{p}(x y)$ in terms of the various $\lambda^{r}(x)$ and $\lambda^{s}(y)$.

A little more precisely, for each relation $\lambda^{p}\left(r_{i}\right)=P_{i, p}$ where $P_{i, p} \in \mathbb{Z}\left[r_{1}, \ldots, r_{m}\right]$ has degree $\leq 1$ and positive coefficients, we obtain a corresponding element $L_{i, p} \in A_{G}^{*}$ which lies in $\operatorname{ker} \pi$ as follows: compute the total Stiefel-Whitney class of $\lambda^{p}\left(r_{i}\right)$ in $A_{G}^{\times}$acording to the rule above, then compute the total StiefelWhitney class of $P_{i, p}$, and call $L_{i, p}$ the difference between the two (viewed as elements of $A_{G}^{*}$ ).

Consider then a presentation

$$
R_{\mathbb{R}}(G)=\mathbb{Z}\left[r_{i}, \lambda^{p} r_{i} \mid 1 \leq i \leq m, 1 \leq p \leq n_{i}\right] / \mathfrak{b} .
$$


Note that we may combine the formulae for tensor products and exterior powers, and "translate" any relation in $\mathfrak{b}$ into a relation in ker $\pi$. The details should be clear by now. Then one has (with $x_{i}$ as in the previous lemma):

Lemma 2.3 - For an element $b \in \mathfrak{b}$, call $R$ the "translation" of $b=0$ into an element of $A_{G}^{*}$, following the rules above for tensor products and exterior powers. Then $R$ is in the ideal generated by the the homogeneous parts of the elements $L_{i, p}$ and $T\left(x_{i}\right)$.

Proof. If we substitute $P_{i, p}$ for $\lambda^{p}\left(r_{i}\right)$ into $b$, we get a polynomial in $\mathbb{Z}\left[r_{1}, \ldots, r_{m}\right]$ which evaluates to 0 in $R_{\mathbb{R}}(G)$, that is, an element of $\mathfrak{a}$. So $b$ can be written as the sum of an element of $\mathfrak{a}$ and an element in the ideal generated by the elements $\lambda^{p}\left(r_{i}\right)-P_{i, p}$. The result now follows easily by an argument as in the previous proof.

Remark 2.4. The reader who feels uncomfortable with the details of lemma 2.2 and 2.3 will be reassured to know that we do not use them in the sequel, strictly speaking. They motivate our decision to give priority to the elements $T\left(x_{i}\right)$ and $L_{i, p}$, but this could have been presented as an arbitrary decision without breaking the logic.

EXAMPLE 2.5 - We return to the example of $G=Q_{8}$ already considered in the introduction. This group has three 1-dimensional, irreducible, real representations $r_{1}, r_{2}$ and $r_{3}$, and an irreducible, 4-dimensional, real representation $\Delta$ of quaternion type. We have $r_{3}=r_{1} r_{2}$ which, as above, yields $w_{1}\left(r_{3}\right)=w_{1}\left(r_{1}\right)+w_{1}\left(r_{2}\right)$.

However, we also have

$$
\lambda^{2}(\Delta)=r_{1}+r_{2}+r_{3}+3 .
$$

Computing the Stiefel-Whitney classes of $\lambda^{2}(\Delta)$ using the formula for exterior powers, together with the fact that $w_{1}(\Delta)=w_{2}(\Delta)=w_{3}(\Delta)=0$ since $\Delta$ has quaternion type, yields in particular $w_{2}\left(\lambda^{2}(\Delta)\right)=0$. On the other hand, one finds that $w_{2}\left(r_{1}+r_{2}+r_{3}+3\right)=w_{1}\left(r_{1}\right) w_{1}\left(r_{2}\right)+w_{1}\left(r_{1}\right) w_{1}\left(r_{3}\right)+w_{1}\left(r_{2}\right) w_{1}\left(r_{3}\right)$, and so this element must be zero. Combined with the expression for $w_{1}\left(r_{3}\right)$, this yields $w_{1}\left(r_{1}\right)^{2}+w_{1}\left(r_{2}\right)^{2}+w_{1}\left(r_{1}\right) w_{1}\left(r_{2}\right)=0$.

Examining the classes in degree 3 rather than 2 gives, similarly, that

$$
w_{1}\left(r_{1}\right)^{2} w_{1}\left(r_{2}\right)+w_{1}\left(r_{1}\right) w_{1}\left(r_{2}\right)^{2}=0 .
$$

The relations obtained in degree 1 and 4 are redundant.

In other words, we have found the following elements in $\operatorname{ker} \pi$ :

$$
\begin{gathered}
\bar{w}_{1}(\Delta), \bar{w}_{2}(\Delta), \bar{w}_{3}(\Delta), \bar{w}_{1}\left(r_{3}\right)-\bar{w}_{1}\left(r_{1}\right)-\bar{w}_{1}\left(r_{2}\right), \\
\bar{w}_{1}\left(r_{1}\right)^{2}+\bar{w}_{1}\left(r_{2}\right)^{2}+\bar{w}_{1}\left(r_{1}\right) \bar{w}_{1}\left(r_{2}\right), \bar{w}_{1}\left(r_{1}\right)^{2} \bar{w}_{1}\left(r_{2}\right)+\bar{w}_{1}\left(r_{1}\right) \bar{w}_{1}\left(r_{2}\right)^{2} .
\end{gathered}
$$

Again in this example, it turns out that $\operatorname{ker} \pi$ is generated by these elements. 


\subsection{Chern classes}

Everything which we have done so far can also be done with Chern rather than Stiefel-Whitney classes, with minor modifications. Moreover, one can draw consequences on Stiefel-Whitney classes by looking at Chern classes, and some of this information cannot be got otherwise. We proceed to explain this.

Let $\rho_{1}, \ldots, \rho_{s}$ denote the complex, irreducible representations of $G$, and let $d_{i}$ be the complex dimension of $\rho_{i}$. There is a universal ring $A_{\mathbb{C}, G}^{*}$ which is polynomial on generators $\bar{c}_{j}\left(\rho_{i}\right)$ for $1 \leq i \leq s$ and $1 \leq j \leq d_{i}$. There is a map $\sigma: A_{\mathbb{C}, G}^{*} \rightarrow H^{*}(G)$, and the image of $\bar{c}_{j}\left(\rho_{i}\right)$ is $c_{j}\left(\rho_{i}\right)$, the $j$-th Chern class of $\rho_{i}$. One can take $A_{\mathbb{C}, G}^{*}$ to be a tensor product of cohomology rings of various classifying spaces of unitary groups, and $g$ is induced by a collection of group homomorphisms.

We write $\mathcal{C}^{*}(G)$ for $A_{\mathbb{C}, G}^{*} / \operatorname{ker} \sigma$, and see it as the subring of $H^{*}(G)$ generated by all Chern classes. A quotient of $A_{\mathbb{C}, G}^{*}$ by an ideal contained in $\operatorname{ker} \sigma$ will be called a formal ring of Chern classes. We obtain examples of formal rings by using the formal properties of Chern classes, which are identical to those of Stiefel-Whitney classes: one only has to bear in mind that $c_{j}\left(r_{i}\right)$ has degree $2 j$ and that the "roots" of the splitting principle have degree 2. Otherwise the formulae for tensor products and exterior powers are the same.

Now, an element in $\operatorname{ker} \sigma$ yields an element in $\operatorname{ker} \pi$ according to the following recipe. If $\rho_{i}$ is the complexification of a real (and irreducible) representation $r$, then one has $c_{j}\left(\rho_{i}\right)=w_{j}(r)^{2}$. Note that $r$ is of real type in this case. If on the other hand, $\rho_{i}$ is not such a complexification, then we let $r$ denote its realification: it is still irreducible, and of either complex or quaternion type. In this case one has $c_{j}\left(\rho_{i}\right)=w_{2 j}(r)$ (while the odd-degree Stiefel-Whitney classes of $r$ are zero, as already pointed out). As a result, if we formally replace each element $\bar{c}_{j}\left(\rho_{i}\right)$ by either $\bar{w}_{j}(r)^{2}$ or $\bar{w}_{2 j}(r)$ following this rule, then indeed any element in $\operatorname{ker} \sigma$ is turned into an element in $\operatorname{ker} \pi$.

It is perhaps as well to say that we have just described a map

$$
\phi: A_{\mathbb{C}, G}^{*} \rightarrow A_{G}^{*}
$$

such that $\sigma=\pi \circ \phi$. It must carry $\operatorname{ker} \sigma$ into $\operatorname{ker} \pi$.

\subsection{Steenrod operations}

The $\operatorname{ring} A_{G}^{*}$ is naturally an unstable algebra, so we have operations $S q^{k}$ for $k \geq 0$ on it. Of course $H^{*}(G)$ is also an unstable algebra, and $\pi$ is compatible with the operations. As a result, the ideal $\operatorname{ker} \pi$ is stable under the Steenrod operations.

Now given any ideal $I$ in $A_{G}^{*}$, there is a unique smallest ideal $S q(I)$ containing it and stable under each $S q^{k}$ (namely the intersection of all such ideals). If $I \subset \operatorname{ker} \pi$, then $S q(I) \subset \operatorname{ker} \pi$.

It is easy to compute $S q(I)$ concretely. If $I$ is generated by $t_{1}, t_{2}, \ldots, t_{\ell}$, then either $I$ is "Steenrod stable" or the ideal $I_{2}$ generated by all elements $S q^{k} t_{i}\left(1 \leq i \leq \ell\right.$ and $\left.0 \leq k<\left|t_{i}\right|\right)$ is strictly bigger than $I$. If $I_{2}$ is not Steenrod stable, we get a strictly bigger ideal $I_{3}$ in the same fashion, and so on. Because $A_{G}^{*}$ is noetherian, this process has to stop, and we obtain $S q(I)$ in finite time. 


\subsection{The ring $\mathcal{W}_{F}^{*}(G)$}

We shall now describe a particular formal ring of Stiefel-Whitney classes, to be denoted $\mathcal{W}_{F}^{*}(G)$, which combines all the relations which we have been discussing.

We proceed as follows:

- First, we let $I \subset \operatorname{ker} \pi$ denote the ideal generated by all the elements $T\left(x_{i}\right)$ and $L_{i, p}$ as in the lemmas 2.2 and 2.3, together with all the "rationality" relations. In other words, we consider all the relations in $\operatorname{ker} \pi$ which are discussed in section 2.2.

- Similarly, we define $J \subset \operatorname{ker} \sigma$ using the relations coming from the tensor product formula and the exterior power formula, only with Chern rather than Stiefel-Whitney classes.

- Next, we consider the ideal $I^{\prime}$ generated by $I$ and $\phi(J)$ (see $\S 2.3$ ).

- Finally, we take $I^{\prime \prime}=S q\left(I^{\prime}\right)$ as in $\S 2.4$. We define

$$
\mathcal{W}_{F}^{*}(G)=A_{G}^{*} / I^{\prime \prime} .
$$

There is a surjective map $\mathcal{W}_{F}^{*}(G) \rightarrow \mathcal{W}^{*}(G)$. Composing it with the inclusion into $H^{*}(G)$ induced by $\pi$, we obtain a map $a: \mathcal{W}_{F}^{*}(G) \rightarrow H^{*}(G)$.

Proposition 2.6 - The map a is an isomorphism in degree 1 , and turns $H^{*}(G)$ into a finitely generated $\mathcal{W}_{F}^{*}(G)$-module.

Proof. The first point follows from the isomorphism $H^{1}(G)=H o m(G, \mathbb{Z} / 2 \mathbb{Z})$. For the second point, we embed $G$ into an orthogonal group $O(n)$, and consider the fibration $O(n) / G \rightarrow B G \rightarrow B O(n)$. Since $O(n)$ is compact, the homogeneous space $O(n) / G$ has finitely many cells, and it follows from the Serre spectral sequence that $H^{*}(G)$ is finitely generated as an $H^{*} B O(n)$-module. $A$ fortiori, it is also finitely generated as a $\mathcal{W}_{F}^{*}(G)$-module.

EXAMPLE 2.7 - Let us consider the group $G$ of order 16 which appears in proposition 1.2. As indicated, this group is the only semidirect product $\mathbb{Z} / 8 \rtimes$ $\mathbb{Z} / 2$ whose centre is cyclic of order 4 . The nonzero element in the $\mathbb{Z} / 2$ factor acts on the $\mathbb{Z} / 8$ factor by multiplication by 5 .

There are 10 conjugacy classes, and so 10 complex, irreducible representations. Leaving out the trivial one, the character table looks like this:

\begin{tabular}{|c|c|c|c|c|c|c|c|c|c|c|}
\hline Conjugacy class & 1 & 2 & 3 & 4 & 5 & 6 & 7 & 8 & 9 & 10 \\
\hline$\rho_{1}$ & 1 & -1 & 1 & 1 & 1 & -1 & -1 & 1 & 1 & -1 \\
$\rho_{2}$ & 1 & 1 & -1 & 1 & 1 & -1 & 1 & -1 & 1 & -1 \\
$\rho_{3}$ & 1 & -1 & -1 & 1 & 1 & 1 & -1 & -1 & 1 & 1 \\
$\rho_{4}$ & 1 & $i$ & 1 & -1 & 1 & $i$ & $-i$ & -1 & -1 & $-i$ \\
$\rho_{5}$ & 1 & $-i$ & 1 & -1 & 1 & $-i$ & $i$ & -1 & -1 & $i$ \\
$\rho_{6}$ & 1 & $i$ & -1 & -1 & 1 & $-i$ & $-i$ & 1 & -1 & $i$ \\
$\rho_{7}$ & 1 & $-i$ & -1 & -1 & 1 & $i$ & $i$ & 1 & -1 & $-i$ \\
$\rho_{8}$ & 2 & 0 & 0 & $2 i$ & -2 & 0 & 0 & 0 & $-2 i$ & 0 \\
$\rho_{9}$ & 2 & 0 & 0 & $-2 i$ & -2 & 0 & 0 & 0 & $2 i$ & 0 \\
\hline
\end{tabular}


Here we have ordered the conjugacy classes arbitrarily (in fact, we follow the choices made by the GAP computer package). The first is the class of the unit in $G$, and the sizes of the classes are 1,2, 2, 1, 1, 2, 2, 2, 1, 2 .

This is enough to compute the rationality according to the recipe in [24] (Prop. 39). We find that the first three representations are the complexifications of $r_{1}, r_{2}, r_{3}$, which have thus real type. The others give irreducible, real representations of complex type after "realification". We let $r_{4}, r_{6}$ and $r_{8}$ be the real representations underlying $\rho_{4}, \rho_{6}$ and $\rho_{8}$ respectively (these are conjugated to $\rho_{5}, \rho_{7}$ and $\rho_{9}$ respectively). The irreducible, real representations of $G$ are exactly $r_{1}, r_{2}, r_{3}, r_{4}, r_{6}, r_{8}$ together with the trivial representation.

Let us explore some of the relations in $\mathcal{W}_{F}^{*}(G)$. From now on, the elements in this ring will be written $w_{j}\left(r_{i}\right)$ rather than $\bar{w}_{j}\left(r_{i}\right)$, for simplicity.

We find that $r_{1}=r_{2} \otimes r_{3}$, so that $w_{1}\left(r_{1}\right)+w_{1}\left(r_{2}\right)+w_{1}\left(r_{3}\right)=0$. Next, we observe that $\lambda^{2}\left(r_{8}\right)=1+r_{1}+r_{2}+r_{3}+r_{6}$ which, taking into account that $w_{1}\left(r_{8}\right)=w_{3}\left(r_{8}\right)=w_{1}\left(r_{6}\right)=0$ because $r_{8}$ and $r_{8}$ have complex type, yields

$$
w_{1}\left(r_{1}\right) w_{1}\left(r_{2}\right) w_{1}\left(r_{3}\right)+w_{2}\left(r_{6}\right)\left[w_{1}\left(r_{1}\right)+w_{1}\left(r_{2}\right)+w_{1}\left(r_{3}\right)\right]=0 .
$$

Combining this with the previous relation, we get

$$
w_{1}\left(r_{2}\right)^{2} w_{1}\left(r_{3}\right)+w_{1}\left(r_{2}\right) w_{1}\left(r_{3}\right)^{2}=0 .
$$

We also note that $\rho_{1}=\rho_{4} \otimes_{\mathbb{C}} \rho_{4}$, so that $c_{1}\left(\rho_{1}\right)=2 c_{1}\left(\rho_{4}\right)=0(\bmod 2)$. However $c_{1}\left(\rho_{1}\right)=w_{1}\left(r_{1}\right)^{2}=w_{1}\left(r_{2}\right)^{2}+w_{1}\left(r_{3}\right)^{2}$. So

$$
w_{1}\left(r_{2}\right)^{2}+w_{1}\left(r_{3}\right)^{2}=0 .
$$

As it turns out, these are all the relations that we shall keep, for we have

$$
\mathcal{W}_{F}^{*}(G)=\frac{\mathbb{F}_{2}\left[w_{1}\left(r_{2}\right), w_{1}\left(r_{3}\right), w_{4}\left(r_{8}\right)\right]}{(R, S)} .
$$

The end of the proof of this is a lengthy exercise for the reader. It involves showing the following relations:

$$
\begin{gathered}
w_{2}\left(r_{4}\right)=w_{1}\left(r_{2}\right)^{2}+w_{1}\left(r_{2}\right) w_{1}\left(r_{3}\right), \quad w_{2}\left(r_{6}\right)=w_{1}\left(r_{2}\right) w_{1}\left(r_{3}\right), \\
w_{2}\left(r_{8}\right)=w_{1}\left(r_{2}\right) w_{1}\left(r_{3}\right) .
\end{gathered}
$$

This explains why we keep only the three variables above in $\mathcal{W}_{F}^{*}(G)$. Also, one should prove that all the other relations that one throws into $\mathcal{W}_{F}^{*}(G)$ are redundant at this point, which of course takes a lot of time (and was done with the help of a computer).

\section{§3. The main algorithm}

In this section we explain in details the procedure outlined in the introduction.

\subsection{Notations \& Preliminaries}

$\diamond$ Choice of variables. We shall assume that we have for $H^{*}(G)$ a presentation in terms of variables $g_{1}, g_{2}, \ldots$ and relations. 
As for $\mathcal{W}_{F}^{*}(G)$, we have a canonical choice of variables which are all of the form $\bar{w}_{j}\left(r_{i}\right)$, and we have computed the relations between these which define $\mathcal{W}_{F}^{*}(G)$ in the previous section. We shall split the variables into three sets, and we shall use the following rule.

Assume that $R$ is a ring with a surjective map $P: k\left[X_{1}, \ldots, X_{n}\right] \rightarrow R$, where $k$ is any field, and let $x_{i}=P\left(X_{i}\right)$, for each $i$. Then we shall say that $x_{i}$, for lack of a better name, is a polynomial variable with respect to this presentation if there is a generating set for ker $P$ which consists of polynomials not involving $X_{i}$. For definiteness, let us rephrase this. Starting with any generating set for an ideal, one may compute the reduced Grobner basis for this ideal using Buchberger's algorithm (see [4]) and this is unique. It is apparent that Buchberger's algorithm does not introduce new variables, and therefore, a variable $x_{i}$ is polynomial if and only if $X_{i}$ does not appear in any of the elements of the reduced Grobner basis for ker $P$. (It also follows that the order on the power products, which is needed for Buchberger's algorithm, is irrelevant here.)

If $x_{1}, \ldots, x_{m}$ are polynomial variables for $R$ with respect to the presentation $P(m \leq n)$, then one has, putting $S=R /\left(x_{1}, \ldots, x_{m}\right)$, the isomorphism $R=$ $S\left[x_{1}, \ldots, x_{m}\right]$.

We apply this to $\mathcal{W}_{F}^{*}(G)$ and its presentation as a quotient of $A_{G}^{*}$. We shall write $t_{1}, t_{2}, \ldots$ for the degree 1 variables. As for the other variables, we write $p_{1}, p_{2}, \ldots$ for those which are polynomial, and $q_{1}, q_{2}, \ldots$ for the others.

Write $\Omega=\mathbb{F}_{2}\left[t_{1}, t_{2}, \ldots, q_{1}, q_{2}, \ldots\right]$, a subring of $\mathcal{W}_{F}^{*}(G)$. Then one has

$$
\mathcal{W}_{F}^{*}(G)=\Omega\left[p_{1}, p_{2}, \ldots\right]
$$

Concretely, we shall compute the reduced Grobner basis for ker $\pi$, and extract from it a minimal set of generators $R_{1}, R_{2}, \ldots$ for this ideal. The variables not showing up in any $R_{k}$ are the polynomial variables (note that some of the $t_{i}$ 's may well be polynomial, too).

EXAMPLE 3.1 - Throughout this section, we shall follow the example of the group $G$ already considered in proposition 1.2 and example 2.7. The algorithm is particularly simple in this case, yet it seems to illustrate most of the features of the general case.

A presentation of $H^{*}(G)$ is as a quotient of the graded polynomial ring $\mathbb{F}_{2}[z, y, x, w]$ with $|z|=|y|=1,|x|=3$ and $|w|=4$. The relations are then:

$$
\begin{aligned}
& z^{2}=0, \quad z y^{2}=0, \\
& z x=0, \quad x^{2}=0 .
\end{aligned}
$$

(These form a Grobner basis.)

On the other hand, as already mentioned, we find that $\mathcal{W}_{F}^{*}(G)$ is a quotient of the polynomial ring $\mathbb{F}_{2}\left[w_{1}\left(r_{2}\right), w_{1}\left(r_{3}\right), w_{4}\left(r_{8}\right)\right]$ where the subscript gives the degree, for some representations $r_{2}, r_{3}$ and $r_{8}$. The relations are:

$$
w_{1}\left(r_{2}\right)^{2}+w_{1}\left(r_{3}\right)^{2}=0, \quad w_{1}\left(r_{2}\right)^{3}+w_{1}\left(r_{2}\right)^{2} w_{1}\left(r_{3}\right)=0 .
$$

Again these form a Grobner basis.

The variable $w_{4}\left(r_{8}\right)$ is polynomial; there is no variable corresponding to the $q_{i}$ 's in this case. 
$\diamond$ Admissible maps; equivalent maps. An admissible map $f: \mathcal{W}_{F}^{*}(G) \rightarrow$ $H^{*}(G)$ is one which is an isomorphism in degree 1 and which turns $H^{*}(G)$ into a finitely generated $\mathcal{W}_{F}^{*}(G)$-module.

When $A$ is a graded $\mathbb{F}_{2}$-algebra, we let $A^{>0}$ denote the ideal of elements of positive degree. If $f: A \rightarrow B$ is any map of graded algebras, we write $\langle f\rangle$ for the ideal of $B$ generated by $f\left(A^{>0}\right)$. When $A$ and $B$ are connected, then $f$ is surjective if and only if $\langle f\rangle=B^{>0}$. Also, $B$ is a finitely generated $A$-module if and only if $B /\langle f\rangle$ is finite dimensional over $\mathbb{F}_{2}$.

Two maps $f$ and $g$ from $\mathcal{W}_{F}^{*}(G)$ to $H^{*}(G)$ are said to be equivalent when they have the same kernel, and when $\langle f\rangle=\langle g\rangle$. This defines an equivalence relation on the set of all maps from $\mathcal{W}_{F}^{*}(G)$ to $H^{*}(G)$.

\subsection{Construction of certain maps $\mathcal{W}_{F}^{*}(G) \rightarrow H^{*}(G)$.}

The main idea is to construct all maps from $\mathcal{W}_{F}^{*}(G)$ to $H^{*}(G)$, then reject those which are not admissible, then reject more maps using finer criteria, and finally hope that the remaining maps are all equivalent. However, we cannot quite follow this programme, for the computation of all maps between these two rings would simply take too much time. Careful precautions will allow us to reduce the number of computations by many orders of magnitude. Some work will be needed to prove that we get a correct answer nonetheless.

In this section, $f$ is a homomorphism $\mathcal{W}_{F}^{*}(G) \rightarrow H^{*}(G)$ which we gradually build by specifying the values $f\left(t_{i}\right)$, then $f\left(q_{i}\right)$, and then $f\left(p_{i}\right)$, step by step.

$\diamond$ Step 1 : setting the degree 1 variables. We start by listing all the possible values for the various $f\left(t_{i}\right)$, that is, we list all choices of $f\left(t_{1}\right), f\left(t_{2}\right), \ldots$ such that

1. $f$ is an isomorphism in degree 1 ,

2. the relations $R_{k}$ involving the elements $t_{i}$ 's only are "satisfied", that is, map to 0 under $f$.

We do this by simply exhausting all elements in degree 1 in $H^{*}(G)$, though we use the following trick in order to save time in the sequel. Whenever we have two possible choices $f$ and $f^{\prime}$, that is whenever we have $a_{1}=f\left(t_{1}\right), a_{2}=f\left(t_{2}\right), \ldots$ on the one hand and $b_{1}=f^{\prime}\left(t_{1}\right), b_{2}=f^{\prime}\left(t_{2}\right), \ldots$ on the other hand such that both conditions are satisfied, we compare them thus: we check whether the map $\alpha: H^{*}(G) \rightarrow H^{*}(G)$ sending $a_{i}$ to $b_{i}$ and all other variables in $H^{*}(G)$ to themselves is well-defined. If so, it is an automorphism of $H^{*}(G)$ such that $f^{\prime}=\alpha \circ f$. Clearly in this case, continuing the process with $f$ or $f^{\prime}$ is immaterial for what follows.

So we keep only one map out of the pair $\left(f, f^{\prime}\right)$. When this is over, we have a set of partially defined maps; for each one, we move to the next step. We keep writing $f$ for a particular choice.

EXAMPLE 3.2 - Resuming example 3.1, we have only one possibility for $f$ after Step 1 , in this case, namely:

$$
f\left(w_{1}\left(r_{2}\right)\right)=z+y, \quad f\left(w_{1}\left(r_{3}\right)\right)=y .
$$

One could have exchanged the roles of $w_{1}\left(r_{2}\right)$ and $w_{1}\left(r_{3}\right)$, but then the automorphism $\alpha$ of $H^{*}(G)$ which sends $y$ to $y+z$ and all other variables to themselves 
would bridge the two options. So we have indeed a single $f$ that we take to the next step.

In passing note that, from the above, we see that there is more symmetry in choosing $w_{1}\left(r_{2}\right)$ and $w_{1}\left(r_{3}\right)$, rather than $y$ and $z$, as the generators in degree 1 .

$\diamond$ Step 2 : setting the value of $f\left(q_{i}\right)$. We wish to continue in the same fashion, and find all possible values for $f\left(q_{i}\right)$. Here "possible values" means that the remaining relations $R_{k}$, not yet considered in step 1, must be satisfied. Again we proceed by exhaustion, but a simple observation can save us a spectacular amount of time.

Instead of defining all $f\left(q_{i}\right)$ 's and then check whether the relations are satisfied, we proceed one relation at a time (of course). Given a relation $R_{k}$ involving $q_{i_{1}}, \ldots, q_{i_{n}}$ as well as degree 1 variables, we find all values for $f\left(q_{i_{1}}\right), \ldots, f\left(q_{i_{n}}\right)$ such that $f\left(R_{k}\right)=0$. Then we move to the next relation.

However, the order in which we consider the relations is crucial. Indeed, suppose that $q_{i_{j}}$ has degree $d_{i_{j}}$, and that $H^{*}(G)$ is of dimension $c_{i_{j}}$ in degree $d_{i_{j}}$. Then if $c=\sum_{j} c_{i_{j}}$, we have $2^{c}$ possibilities for the values of the variables $q_{i_{j}}$. This number $2^{c}$ we call the weight of $R_{k}$. We start our investigation with the relation of lowest weight. Then, having made a choice for $f\left(q_{i_{j}}\right)$, we recompute the weights of the other relations, which have decreased because there are now fewer choices to make. We proceed with the lowest weight relation remaining, and so on.

Looking for the possibilities in this order rather than a random order can reduce the computing time from hours to minutes.

Of course it may happen, for a given $f$ resulting from Step 1, that there is no way of completing Step 2. However, the existence of the map $a$ guarantees that at least one choice can pass both steps. Let $f$ be such a map, defined on $\Omega$.

$\diamond$ Step 3 : Setting the value of $f\left(p_{i}\right)$. When we come to the polynomial variables, any value for $f\left(p_{i}\right)$ gives a well defined homomorphism $f$. However, in practical terms, this means that the number of homomorphisms that we end up with is simply too large: finishing the algorithm would take far too much time. Instead we use the following simplification, which slightly increases the chances of failure of the algorithm but greatly improves the speed. (Although as we point out later, if one is particularly interested in a single group $G$ and is willing to wait long enough, it may be best not to use this trick).

Let $R$ denote the quotient of $H^{*}(G)$ by the ideal generated by $f\left(\Omega^{>0}\right)$ (in the notation above this is $R=H^{*}(G) /\langle f\rangle$ if one keeps in mind that $f$ is only defined on $\Omega$ so far). We extend the composition $\bar{f}: \Omega \rightarrow H^{*}(G) \rightarrow R$ to a map $\bar{f}: \mathcal{W}_{F}^{*}(G) \rightarrow R$ by choosing $\bar{f}\left(p_{i}\right)$ arbitrarily (but of the right homogeneous degree, of course). The point being that $R$ is much smaller than $H^{*}(G)$ and there are relatively few choices for $\bar{f}$.

Then we pick an arbitrary lift for $\bar{f}$, giving finally a map $f: \mathcal{W}_{F}^{*}(G) \rightarrow$ $H^{*}(G)$. Note that any two lifts $f$ and $f^{\prime}$ have $\langle f\rangle=\left\langle f^{\prime}\right\rangle$. In particular, the finite generation of $H^{*}(G)$ as a module over $\mathcal{W}_{F}^{*}(G)$ via the map $f$ does not depend on the choice of lift.

EXAMPLE 3.3 - We continue with the $f$ of example 3.2. The dimension of $H^{4}(G)$ is 3 , with a basis given by $y^{4}, y x$ and $w$ for example. So we have $2^{3}=8$ 
choices for $f\left(w_{4}\left(r_{8}\right)\right)$. However, the ring $R$ is generated by (the images of) $x$ and $w$, so that it is 1-dimensional in degree 4 (with $w$ the only nonzero element in this degree), which leaves only 2 choices for $\bar{f}$. We end up with two possibilities for $f$ : we may either send $w_{4}\left(r_{8}\right)$ to 0 , or to $w$ (in either case, any other lift would do, but we keep only one).

This is a toy example of course, and the computer could well exhaust all 8 possibilities. However, we point out that dividing the number of subsequent computations by 4 is quite satisfactory, and such reductions become inevitable if one wishes to deal with bigger groups (the sensitivity being exponential).

\subsection{Tests \& Conclusions}

We have now a certain finite set $S$ of maps $\mathcal{W}_{F}^{*}(G) \rightarrow H^{*}(G)$. We shall now run a series of tests on the maps in $S$, leading either to definite conclusions regarding the map $a$, or to the decision to give up on the computation.

At this point it is not clear whether $a \in S$, or even whether $a$ is equivalent to a map in $S$. However, there is certainly a map $f$ in $S$ which agrees with $a$ on $\Omega$ (perhaps with the degree 1 variables in $H^{*}(G)$ relabelled, cf Step 1), and with $\langle a\rangle=\langle f\rangle$.

$\diamond$ Test 1 : finite generation of $H^{*}(G)$. We reject all the maps in $S$ which are not admissible, ie those which do not turn $H^{*}(G)$ into a finitely generated $\mathcal{W}_{F}^{*}(G)$-module. There remains a smaller set $S^{\prime}$. The last remark shows that $S^{\prime}$ is not empty.

EXAMPLE 3.4 - We continue from example 3.3. Out of our two maps in $S$, only one is admissible, namely that with $f\left(w_{4}\left(r_{8}\right)\right)=w$. Thus we keep only this $f$.

$\diamond$ Test 2 : polynomial variables. Let $f \in S^{\prime}$. We check which of the given generators for $H^{*}(G)$ are non zero in $H^{*}(G) /\langle f\rangle$; for simplicity, say these are numbered $g_{1}, \ldots, g_{m}$. We adjoin polynomial variables with the same name to $\mathcal{W}_{F}^{*}(G)$, thus obtaining

$$
\mathcal{W}_{F}^{*}(G)^{+}=\mathcal{W}_{F}^{*}(G)\left[g_{1}, \ldots, g_{m}\right] .
$$

The map $f$ has an obvious extension to $\mathcal{W}_{F}^{*}(G)^{+}$which we call $f^{+}$; it is surjective.

We then compute the reduced Grobner basis of $\operatorname{ker}\left(f^{+}\right)$. If the polynomial variables $p_{i}$ show up in this Grobner basis, that is if the $p_{i}$ 's are not polynomial anymore in $\mathcal{W}_{F}^{*}(G)^{+} / \operatorname{ker}\left(f^{+}\right)$with respect to the obvious presentation, we give up on the computation altogether. Otherwise, if all maps in $S^{\prime}$ pass this test, we move to Test 3 with $S^{\prime}$ unchanged.

We shall give below a heuristic explanation according to which it is reasonable to expect that Test 2 is often completed succesfully. Our interest in this test comes from the following lemma.

Lemma 3.5 - Let $f \in S^{\prime}$ satisfy the test above, and let $g$ be a map $\mathcal{W}_{F}^{*}(G) \rightarrow$ $H^{*}(G)$ obtained by a different choice of lift in Step 3. Then $f$ and $g$ are equivalent. Also, $f^{+}$and $g^{+}$are equivalent. 
Proof. We have pointed out that $\langle f\rangle=\langle g\rangle$ always, so we need to show that $\operatorname{ker} f=\operatorname{ker} g$.

Assume that the $p_{i}$ 's are ordered by degree, that is assume that $\left|p_{1}\right| \leq\left|p_{2}\right| \leq$ $\ldots$. Write $\Omega^{+}=\Omega\left[g_{1}, \ldots, g_{m}\right]$ so that $\mathcal{W}_{F}^{*}(G)^{+}=\Omega^{+}\left[p_{1}, p_{2}, \ldots\right]$. Note that $f^{+}$and $g^{+}$are both defined on this ring $\mathcal{W}_{F}^{*}(G)^{+}$, and are both surjective. We define an automorphism $\alpha$ of $\mathcal{W}_{F}^{*}(G)^{+}$such that $f^{+}=g^{+} \circ \alpha$.

Indeed, since $g^{+}$is surjective, and from the definitions, we see that

$$
f^{+}\left(p_{i}\right)=f\left(p_{i}\right)=g\left(p_{i}\right)+g^{+}\left(\omega_{i}\right)=g^{+}\left(p_{i}+\omega_{i}\right)
$$

for some $\omega_{i} \in \Omega^{+}\left[p_{j}:\left|p_{j}\right|<\left|p_{i}\right|\right]$. So we may define $\alpha$ by requiring it to be the identity on $\Omega^{+}$, and to send $p_{i}$ to $p_{i}+\omega_{i}$. In order to see that $\alpha$ is an isomorphism, one may for example show by induction on $i$ that $\omega_{i}$, and thus $p_{i}$, is in the image of $\alpha$; therefore $\alpha$ is surjective and is an isomorphism as a result.

It is now easy to conclude. Let $b_{1}, b_{2}, \ldots$ be the reduced Grobner basis for $\operatorname{ker}\left(f^{+}\right)$. By choosing the term order carefully, we can arrange things so that the $b_{i}$ 's not involving the variables $g_{1}, \ldots, g_{m}$ constitute the reduced Grobner basis for ker $f$; say these are $b_{1}, \ldots, b_{r}$. Now, since $f$, or rather $f^{+}$, passes Test 2 , then the elements $b_{1}, \ldots, b_{r}$ do not involve the $p_{i}$ 's, either. It follows that $\alpha\left(b_{i}\right)=b_{i}$ and that $\operatorname{ker} f \subset \operatorname{ker} g$.

From the relation $g^{+}=f^{+} \circ \alpha^{-1}$, it is clear that $g$ passes Test 2 as well, so we may reverse the roles and obtain $\operatorname{ker} g \subset \operatorname{ker} f$.

Proving that $f^{+}$and $g^{+}$are equivalent is a similar, but easier, matter.

Assuming that all maps in $S^{\prime}$ have passed the test, we can move on to Test 3 knowing that $a$ is equivalent to some map in $S^{\prime}$.

Example 3.6 - We continue from example 3.4. There is only one $f$ to deal with. In this case $H^{*}(G) /\langle f\rangle$ is generated by $x$ only as an algebra, so we adjoin a variable $x$ to $\mathcal{W}_{F}^{*}(G)$, obtaining $\mathcal{W}_{F}^{*}(G)^{+}$which is generated by $w_{1}\left(r_{2}\right), w_{1}\left(r_{3}\right)$, $w_{4}\left(r_{8}\right)$ and $x$. We extend $f$ to this ring by setting $f^{+}(x)=x$.

A Grobner basis for $\operatorname{ker}\left(f^{+}\right)$is then

$$
\begin{gathered}
w_{1}\left(r_{2}\right)^{2}+w_{1}\left(r_{3}\right)^{2}, \quad w_{1}\left(r_{2}\right)^{2} w_{1}\left(r_{3}\right)+w_{1}\left(r_{2}\right) w_{1}\left(r_{3}\right)^{2}, \\
w_{1}\left(r_{2}\right) x+w_{1}\left(r_{3}\right) x, \quad x^{2} .
\end{gathered}
$$

These do not involve $w_{4}\left(r_{8}\right)$. Test 2 is successful.

Note that, since we have only one map in $S^{\prime}$, there is no need to perform Test 3 and Test 4 , which we describe now in the general case.

$\diamond$ Test 3 : Steenrod operations. We reject all maps in $S^{\prime}$ whose kernel is not stable under the Steenrod operations. There remains a smaller set $S^{\prime \prime}$. Since $a$ is a map of unstable algebras, $S^{\prime \prime}$ is not empty.

$\diamond$ Test 4 : restrictions to elementary abelian subgroups. When $E$ is an elementary abelian 2-group, then $H^{*}(E)$ is completely understood, including Stiefel-Whitney classes. One way to state this is to say that $a_{E}: \mathcal{W}_{F}^{*}(E) \rightarrow$ $H^{*}(E)$ is an isomorphism, and that (as always) the map $A_{E}^{*} \rightarrow \mathcal{W}_{F}^{*}(E)$ is explicitly described.

We exploit this to setup our final test. The map $a: \mathcal{W}_{F}^{*}(G) \rightarrow H^{*}(G)$ can be composed with the restriction $H^{*}(G) \rightarrow H^{*}(E)$ for any elementry abelian 
subgroup $E$ of $G$, thus giving a map $\mathcal{W}_{F}^{*}(G) \rightarrow H^{*}(E)$ which we understand fully: to determine the image of $w_{j}\left(r_{i}\right)$, decompose $r_{i}$ as a sum of irreducible, real representations of $E$, and compute the Stiefel-Whitney of this sum using the usual formula; then use the map $A_{E}^{*} \rightarrow H^{*}(E)$ to express the result in terms of your favorite choice of generators for $H^{*}(E)$.

Our test is the following. If the generators for $\operatorname{ker} f$ do not map to 0 under the restriction maps $\mathcal{W}_{F}^{*}(G) \rightarrow H^{*}(E)$, for $E$ running among the maximal elementary abelian subgroups, then we reject $f$ from $S^{\prime \prime}$. We obtain in this way a smaller, nonempty set $S^{\prime \prime \prime}$.

$\diamond$ Conclusion. If the maps in $S^{\prime \prime \prime}$ are not all equivalent, the computation has failed. If they are, we compute for each $f \in S^{\prime \prime \prime}$ the map $f^{+}$has above; note that all these are defined on the same ring $\mathcal{W}_{F}^{*}(G)^{+}$. If the maps $f^{+}$are not all equivalent, the computation has failed. Otherwise, we claim that we have succeeded, in a sense which we make precise now.

Pick an $f$ in $S^{\prime \prime \prime}$. Then $f$ is equivalent to $a$. Moreover $f^{+}$and $a^{+}$are defined on the same ring $\mathcal{W}_{F}^{*}(G)^{+}$, are both surjective, and are equivalent. Thus, we know the kernel of the surjective map

$$
a^{+}: \mathcal{W}_{F}^{*}(G)\left[g_{1}, \ldots, g_{m}\right] \rightarrow H^{*}(G) .
$$

This is the desired presentation of $H^{*}(G)$.

EXAMPLE 3.7 - We conclude example 3.6, and the proof of proposition 1.2 at the same time. There being only one candidate in $S^{\prime}$, Test 3 , Test 4 and the final check are all redundant. Note that the map $f$ is not necessarily equal to $a$ : in Step 1 we had two choices, and in Step 3 we had four, so we can write down 8 maps from $\mathcal{W}_{F}^{*}(G)$ to $H^{*}(G)$, one of which will be $a$. However, these are all equivalent, and their extensions to $\mathcal{W}_{F}^{*}(G)^{+}$are also all equivalent. In the end, we know the kernel of $a^{+}$, as it was given in the previous example. Thus proposition 1.2 holds (the information on Steenrod operations follows from Wu's formula).

\subsection{Comments}

All of the comments below will have something to do with the trick used in Step 3 and its validation in Test 2 .

$\diamond$ A variant. There is an evident variation that we may want to try: namely, in Step 3, drop the ring $R$ and the choice of lifts altogether, and simply gather all possible homomorphisms by listing all possible values for the polynomial variables. Then Test 2 becomes irrelevant.

As already pointed out, this will often lead to a number of elements in the set $S$ which is impossible to manage: each homomorphism in $S$ will need to have

its kernel computed, and this uses Buchberger's algorithm for Grobner basis, a time-consuming process of exponential complexity. However, in very particular cases, it may still be best to go down this road anyway.

EXAMPLE 3.8 - Consider the group number 12 (in the GAP library) of order 64 ; it can be described as $(\mathbb{Z} / 4 \rtimes \mathbb{Z} / 8) \rtimes \mathbb{Z} / 2$. The algorithm above produces about 60 homomorphisms after Step 3, and Test 2 fails. However, the variant 
algorithm produces 384 homomorphisms, which are all surjective and fall into 9 equivalence classes. They all pass Test 3. Fortunately only one of them passes Test 4, and the computation is complete.

Similarly, we may look at the group number 87 of order 64 , a group of type $\mathbb{Z} / 2 \times((\mathbb{Z} / 8 \times \mathbb{Z} / 2) \rtimes \mathbb{Z} / 2)$. The normal algorithm yields about 800 homomorphisms, and Test 2 fails. It is still possible to use the variant, even though there are now 24,576 homomorphisms to deal with. They are all surjective, fall into 5 equivalence classes, only one of which passes Test 3 . The computation is complete, and takes about 30 minutes on an average computer. Clearly, we cannot let the complexity gain an extra order of magnitude.

$\diamond$ The success of Test 2. There are above 100 groups for which our computations are successful; only 4 of them have required the lengthy alternative algorithm. On the other hand, in the vast majority of cases, when the computation fails it does so for reasons other than Test 2. This means that the test is often passed, and indeed it was our hope that it should be so.

A loose explanation is as follows. The $\operatorname{ring} \mathcal{W}_{F}^{*}(G)$ is sufficiently fine that the kernel of $a$ is relatively small; in particular, if a variable is polynomial in $\mathcal{W}_{F}^{*}(G)$, it is unlikely that its image should not be polynomial in $H^{*}(G)$. So the map $a$ itself should pass Test 2. Now, this tells us something about the size of $H^{*}(G)$ relative to that of $\mathcal{W}_{F}^{*}(G)$, and if another homomorphism $f: \mathcal{W}_{F}^{*}(G) \rightarrow H^{*}(G)$ were to fail Test 2 , that is, were to have a polynomial variable showing up in its kernel, it is likely to have an image which is too small, and thus Test 1 will reject it. Otherwise, $f$ would have to have a much bigger image on $\Omega$ than $a$ does, which again is unlikely.

The examples above show that "unlikely" does not mean "impossible". We also note that a refinement of $\mathcal{W}_{F}^{*}(G)$, which one could obtain by thinking of more relations to throw in, would increase the chances of our algorithm.

$\diamond$ The order of the tests. It is tempting to run the straightforward Test 3 and Test 4 first, and thus have a smaller set of homomorphisms on which to try the more dubious Test 2. However, this cannot be done. Indeed, a map could well fail Test 3, say, whereas another choice of lift in Step 3 would give a map that passes it.

\section{$\S 4 . \quad$ Experimental results}

We shall first comment on the practicalities on the computations, and then on the mathematics.

\subsection{Practicalities}

$\diamond$ The programs. The first task is to gather information on the characters of the group $G$, and on the sizes of the conjugacy classes. From this, one can compute scalar products between characters, and thus express tensor products and exterior powers in terms of the irreducible representations. One also finds out what the real characters are. All this is done with the help of the GAP computer package.

The bulk of our project, comprising more than $99 \%$ of the code, is a $\mathrm{C}++$ program which computes a presentation for $\mathcal{W}_{F}^{*}(G)$ and then goes through the 
algorithm just presented. There are about 18, 000 lines of $\mathrm{C}++$ code in standard presentation, to which one must add about 5,000 lines of comments (by comparison, the $\mathrm{AT}_{\mathrm{E}} \mathrm{X}$ source for the present article has just above 2, 000 lines).

It is also necessary to get the information on $H^{*}(G)$ from Carlson's webpage, which is presented there as a Magma file. In order to download all the necessary files automatically and translate them into $\mathrm{C}++$, we have used the Python programming language. Incidentally, Python was also used to produce the various HTML files containing the results.

It has been very convenient to use the SAGE computer package, which allows the smooth blending of GAP, Python, and $\mathrm{C}++$.

$\diamond$ The computing time. All computations were performed on the irmasrv3 server at the university of Strasbourg. This machine has 12 CPUs, which was extremely handy to run the various calculations in parallel. Each CPU though has the power of a standard, personal machine.

The preliminaries, before the algorithm of section 3 starts, take little time. It may happen that the computation of universal polynomials, used in the formulae for tensor products for example as in section 2, take several minutes.

The main algorithm can in many cases be completed in a few seconds; sometimes it can take above 20 minutes (group 87 of order 64 ); or it can take several hours (for example for $Q_{8} \times(\mathbb{Z} / 2)^{3}$, for which it is of course preferable to use the Kunneth formula).

Also, occasionally, the algorithm seems to take so long that we have interrupted it and given up on the computation. The reader may be surprised to learn that it is mostly innocent-looking Step 2 which is particularly timeconsuming. This is in fact the most common cause of failure of the algorithm, much more frequently encountered than a failure after Test 2 or at the very end when there are more than one equivalence class.

\subsection{Mathematical results}

$\diamond$ Success. As announced in the introduction, we have focused on the groups of order dividing 64 . The computation was successful for the 5 groups of order 8 , for 13 of the 14 groups of order 16 , for 28 of the 51 groups of order 32 , and for 61 of the 267 groups of order 64 (a total of 107 groups).

Note that the method is not well-behaved with respect to products: even if we can successfully run the computation for both $G$ and $H$, it may still fail for $G \times H$ (because the complexity explodes).

$\diamond$ Cohomology rings generated by Stiefel-Whitney classes. Among our succesful computations, only 13 groups have been found to have a cohomology which is not generated by Stiefel-Whitney classes. Of course one may argue that the algorithm is more likely to terminate without incident when the cohomology is generated by such classes (and there are no maps $f^{+}$to consider at all). However we have pointed out that the main cause of failure is the excessive time needed by the calculations, and so we find it reasonable to conclude that "most" groups have $\mathcal{W}^{*}(G)=H^{*}(G)$. 
$\diamond$ A curiosity: groups with isomorphic cohomologies. Let $G$ be the group of order 32 whose Hall-Senior number is 21 ; its number in the GAP library is 12 , and it can be described as a semidirect product $\mathbb{Z} / 4 \rtimes \mathbb{Z} / 8$. On the other hand, let $G^{\prime}$ be the group of order 32 whose Hall-Senior number is 29 ; its number in GAP is 14 and it is also a semidirect product, this time $\mathbb{Z} / 8 \rtimes \mathbb{Z} / 4$.

Then $H^{*}(G)$ and $H^{*}\left(G^{\prime}\right)$ are isomorphic rings. What is more, our computations show that they are isomorphic as unstable algebras, that is, there is an isomorphism between them which commutes with the Steenrod operations.

This implies classically ([18], Prop. 3.1.5.2) that, for any elementary abelian 2 -group $E$, there exists a bijection $\operatorname{Rep}(E, G)=\operatorname{Rep}\left(E, G^{\prime}\right)$ (the set $\operatorname{Rep}(A, B)$ consists of all group homomorphisms from $A$ into $B$ up to conjugacies in $B$ ).

\section{$\S 5 . \quad$ Application to algebraic cycles}

\subsection{Algebraic cycles in the cohomology}

$\diamond$ The Chow ring. For any algebraic group $G$ over $\mathbb{C}$, for example a finite group, the classifying space $B G$ can be approximated by algebraic varieties, in such a way that there is a well-defined Chow ring $C H^{*} B G$. As the notation suggests, everything works as if $B G$ were an algebraic variety itself, and $C H^{*} B G$ is to be thought of as generated by the subvarieties of $B G$. For details see [26].

There is a cycle map

$$
c l: C H^{*} B G \rightarrow H^{2 *}(B G, \mathbb{Z}),
$$

whose image we denote by $\mathfrak{C h}^{*}(G)$. Cohomology classes in $\mathfrak{C h}^{*}(G)$ are usually said to be supported by algebraic varieties. Our aim is to compute $\mathfrak{C h}^{*}(G)$ for as many groups $G$ as possible, using our results on Stiefel-Whitney classes. More precisely, we will obtain information on the composition

$$
C H^{*} B G \otimes_{\mathbb{Z}} \mathbb{F}_{2} \rightarrow H^{2 *}(B G, \mathbb{Z}) \otimes_{\mathbb{Z}} \mathbb{F}_{2} \rightarrow H^{2 *}\left(B G, \mathbb{F}_{2}\right) .
$$

This map we still denote by $c l$, and its image by $\mathfrak{C h}^{*}(G)$. Also, $C H^{*} B G$ will stand for $C H^{*} B G \otimes_{\mathbb{Z}} \mathbb{F}_{2}$ from now on, unless we repeat the reduction mod 2 for emphasis. Recall that we write $H^{*}(G)$ for the mod 2 cohomology of $G$.

$\diamond$ A lower bound. If $V$ is a complex representation of $G$, then it has Chern classes $c_{i}(V) \in H^{*}(G)$, which are pulled-back from $H^{*}\left(B G L_{n}(\mathbb{C}), \mathbb{F}_{2}\right)$. However, it turns out that the cycle map $c l$ is an isomorphism for $G L_{n}(\mathbb{C})$ (see [26]), so we have an identification $H^{*}\left(B G L_{n}(\mathbb{C}), \mathbb{F}_{2}\right)=C H^{*} B G L_{n}(\mathbb{C})$.

What is more, the cycle map $c l$ is natural in $G$. It follows that the Chern classes $c_{i}(V)$ "come from the Chow ring", ie are in the image of the cycle map for $G$. In symbols,

$$
\mathcal{C}^{*}(G) \subset \mathfrak{C h}^{*}(G)
$$

$\diamond$ An upper bound. The Steenrod algebra acts on the ring $C H^{*} B G \otimes_{\mathbb{Z}} \mathbb{F}_{2}$, and the cycle map commutes with the operations $S q^{k}$ : for this see [6]. However $S q^{1}$ acts trivially. Note that $C H^{*} B G$ is often seen as a graded ring concentrated in even degrees, with $C H^{n} B G$ in degree $2 n$; with this convention, $S q^{k}$ raises the degrees by $k$, so if $k$ is odd then $S q^{k}$ must be zero on the Chow ring indeed. 
Let $\left\langle S q^{1}\right\rangle$ be the two-sided ideal generated by $S q^{1}$ in the mod 2 Steenrod algebra. We see that $\left\langle S q^{1}\right\rangle$ acts as 0 on the Chow ring of any variety; as a result, any class in $\mathfrak{C h}^{*}(G)$ is killed by $\left\langle S q^{1}\right\rangle$.

It is traditional to write $\tilde{\mathcal{O}} H^{*}(G)$ for the subring of $H^{*}(G)$ of all those evendegree classes which are killed by $\left\langle S q^{1}\right\rangle$. This is the largest unstable submodule of $H^{*}(G)$ which is concentrated in even degrees. With this notation one has:

$$
\mathfrak{C h}^{*}(G) \subset \tilde{\mathcal{O}} H^{*}(G) .
$$

\subsection{Computations}

Our strategy is pretty simple-minded: we shall compute $\mathcal{C}^{*}(G)$ and $\tilde{\mathcal{O}} H^{*}(G)$, and hope that they coincide. In such cases (which are quite common, as we shall see), these two subrings also coincide with $\mathfrak{C h}^{*}(G)$.

The ring $\mathcal{C}^{*}(G)$ is trivial to describe for those groups $G$ for which our previous computations were successful: indeed we have explained in $\S 2.3$ how to express Chern classes in terms of Stiefel-Whitney classes, and we have a full understanding of the map $A_{G}^{*} \rightarrow \mathcal{W}^{*}(G)$.

As for $\tilde{\mathcal{O}} H^{*}(G)$, we need a couple of results before we start.

$\diamond$ Milnor derivations. Define $Q_{0}=S q^{1}$ and

$$
Q_{n+1}=S q^{2^{n+1}} Q_{n}+Q_{n} S q^{2^{n+1}}
$$

Then each $Q_{i}$ acts as a derivation on any unstable algebra, and is called the $i$-th Milnor derivation (see [19]). They all commute with each other.

Lemma 5.1 - If $A$ is an unstable algebra and $x \in A$ has even degree, then $x$ belongs to $\tilde{\mathcal{O}} A$ if and only if $Q_{i}(x)=0$ for all $i \geq 0$.

Proof. In fact, let $A^{\prime}$ denote the algebra of all elements (of even degree or not) killed by each $Q_{i}$, and let $A^{\prime \prime}$ denote the algebra of all elements (again, of arbitrary degree) killed by $\left\langle S q^{1}\right\rangle$. We prove that $A^{\prime}=A^{\prime \prime}$.

Since the Milnor derivations are clearly in $\left\langle S q^{1}\right\rangle$, we certainly have $A^{\prime \prime} \subset A^{\prime}$. On the other hand, $Q_{0}=S q^{1}$, so it suffices to show that $A^{\prime}$ is stable under the Steenrod operations to get the inclusion $A^{\prime} \subset A^{\prime \prime}$.

This follows from [19], theorem 4a, from which we extract just one formula:

$$
Q_{k} S q^{r}=\sum_{i=0}^{\infty} S q^{r-2^{k}\left(2^{i+1}-1\right)} Q_{k+i} .
$$

This is really a finite sum, with the convention that $S q^{a}=0$ when $a<0$. Clearly this proves the claim.

$\diamond$ The kernel of a derivation. If $A$ is an algebra over a field $k$, and

$$
d: A \rightarrow A
$$

is a derivation, how are we to compute generators for the algebra ker $d$ ? Here is the simple method which we have used.

We assume that we have a subalgebra $B$ of $A$ such that: 
- $d$ vanishes on $B$. Thus $d$ is $B$-linear when $A$ is viewed as a $B$-module.

- there is a presentation $r_{A}: \tilde{A} \rightarrow A, \operatorname{resp} r_{B}: \tilde{B} \rightarrow B$, where $\tilde{A}$, resp $\tilde{B}$, is a polynomial ring. These are compatible in the sense that there is a commutative diagram

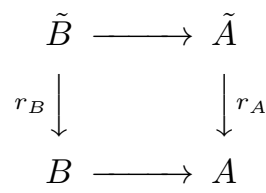

where the horizontal maps are inclusions.

- $\tilde{A}$ is a free $\tilde{B}$-module of finite rank $n$.

In the case at hand, namely finitely generated algebras over $\mathbb{F}_{2}$, it is easy to find such a $B$, mostly because $d$ vanishes on squares. Assume that $A$ is presented as a quotient of $\tilde{A}=\mathbb{F}_{2}\left[X_{i}\right]$, and write $x_{i}=r_{A}\left(X_{i}\right)$. If $d\left(x_{i}\right)=0$, put $Y_{i}=X_{i}$; if not, put $Y_{i}=X_{i}^{2}$. The algebra $\tilde{B}=\mathbb{F}_{2}\left[Y_{i}\right]$ and its quotient $B=\tilde{B} / \operatorname{ker} r_{A}$ together satisfy the properties given. (Note that we could simply take $Y_{i}=X_{i}^{2}$ for all $i$, but this increases the rank $n$, which is not desirable in practice.)

Let us introduce some notations. We let $\tilde{\varepsilon}_{1}, \ldots, \tilde{\varepsilon}_{n}$ be generators for $\tilde{A}$ as a $\tilde{B}$-module. Using these we can and we will identify $\tilde{A}$ and $\tilde{B}^{n}$. Put $\varepsilon_{i}=r_{A}\left(\tilde{\varepsilon}_{i}\right)$. We write $p: \tilde{B}^{n} \rightarrow A$ for the map of $\tilde{B}$-modules underlying $r_{A}$, and we let $\sigma_{1}, \ldots, \sigma_{k}$ be generators for $\operatorname{ker} p$. (When we know generators $f_{1}, f_{2}, \ldots$ for $\operatorname{ker} r_{A}$ as an ideal, then the collection of all elements $\tilde{\varepsilon}_{i} f_{j}$ provides a choice of such generators for $\operatorname{ker} p$ ).

We then pick a lift $\tilde{d}$ of $d$ :

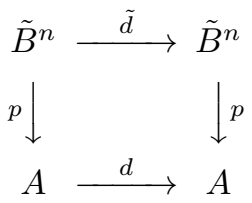

Let $d_{i}=\tilde{d}\left(\tilde{\varepsilon}_{i}\right) \in \tilde{B}^{n}$. Now if $x=\sum b_{i} \varepsilon_{i} \in A$, with each $b_{i} \in B$, then $x$ belongs to ker $d$ if and only if $\sum \tilde{b}_{i} d_{i} \in \operatorname{ker} p$, where $r_{B}\left(\tilde{b}_{i}\right)=b_{i}$. In other words this happens if and only if there exist elements $c_{i} \in \tilde{B}$ such that

$$
\sum_{i=1}^{n} \tilde{b}_{i} d_{i}+\sum_{i=1}^{k} c_{i} \sigma_{i}=0 .
$$

Or, to say this yet differently, the element $\left(\tilde{b}_{1}, \ldots, \tilde{b}_{n}, c_{1}, \ldots, c_{k}\right)$ of $\tilde{B}^{n+k}$ belongs to the syzygy module of the elements $d_{1}, \ldots, d_{n}, \sigma_{1}, \ldots, \sigma_{k}$, which all live in $\tilde{B}^{n}$.

Now, computing generators for the syzygy module of a collection of elements in a free module over a polynomial ring is standard computational algebra ${ }^{1}$, see [4]. Having computing generators, we only keep their first $n$ coordinates (ie we keep the $\tilde{b}_{i}$ 's and drop the $c_{i}$ 's). Applying $p$ yields generators for ker $d$.

\footnotetext{
${ }^{1}$ as is, probably, the computation of the kernel of a derivation. In this paragraph our goal is to justify and explain our own method, in particular to the benefit of readers of the source code. We point out that feeding our results on Steenrod operations to the appropriate sofware would require a considerable amount of work anyway; and more seriously, as we proceed recursively with $d$ playing the role of each $Q_{i}$ one after the other, we have been able to include a number of optimizing tricks, saving work between one computation and the next.
} 
$\diamond$ Computing in finite time. We are now prepared to compute $\operatorname{ker} Q_{i}$ for each $i$. The algebra $\tilde{\mathcal{O}} H^{*}(G)$ is the intersection of all those. The intersection of the first $N+1$ kernels is easy to obtain, for it is the kernel of $Q_{N}$ viewed as a derivation on the algebra recursively computed as the intersection of the first $N$ kernels. (Recall that the Milnor derivations commute).

However, we would like to compute only a finite number of kernels. The next lemma follows from 5.1.

Lemma 5.2 - If the integer $N$ is such that the even part of

$$
\bigcap_{i=0}^{N} \operatorname{ker} Q_{i}
$$

is stable under the Steenrod operations, then this even part is $\tilde{\mathcal{O}} H^{*}(G)$.

This lemma provides an easy test for completion. Moreover:

Proposition 5.3 - There exists an $N$ as in the lemma. In other words, the computation of $\tilde{\mathcal{O}} H^{*}(G)$ terminates in finite time.

Proof. Let $A=H^{*}(G)$, and let $\Omega=\Omega_{A / \mathbb{F}_{2}}$ be the module of $\mathbb{F}_{2}$-differentials of $A$. Then $\operatorname{Der}(A, A)=\operatorname{Hom}_{A}(\Omega, A)$.

If $A$ is generated by elements $x_{1}, \ldots, x_{n}$ as an algebra, then $\Omega$ is generated by the elements $d x_{1}, \ldots, d x_{n}$ as an $A$-module, and in particular it is finitely generated. Thus $\operatorname{Hom}_{A}(\Omega, A)$ injects in a free $A$-module of finite rank, and since $A$ is Noetherian, it follows that $\operatorname{Der}(A, A)$ is finitely generated as an $A$ module.

Thus for all $N$ larger than some $N_{0}$, the derivation $Q_{N}$ is an $A$-linear combination of the derivations $Q_{0}, \ldots, Q_{N_{0}}$, and we see that

$$
\operatorname{ker} Q_{N} \subset \bigcap_{i=0}^{N_{0}} \operatorname{ker} Q_{i}
$$

Therefore we may take $N_{0}$ in the lemma.

\subsection{Results}

$\diamond$ Success and failure. We have attempted to go through the above procedure for all the groups $G$ "at hand", namely all those for which the computation of Stiefel-Whitney classes was completed and for which $H^{*}(G)=\mathcal{W}^{*}(G)$. In such cases the action of the Steenrod operations is already given, while for other groups more work would be needed to find out the action of each $S q^{k}$ on cohomology classes which are not Stiefel-Whitney. So we had $107-13=94$ groups to try (see $\S 4$ ).

In principle there is nothing to prevent the calculation from reaching its end. However in practice, it may happen that the computer runs out of memory, or that the computation takes simply too long. We have obtained answers in the following cases: for all 5 groups of order 8, for 10 groups of order 16, for 17 groups of order 32 , and for 30 groups of order 64 , a total of 62 groups. 
Among these, 38 groups $G$ have $\mathcal{C}^{*}(G)=\mathfrak{C h}^{*}(G)=\tilde{\mathcal{O}} H^{*}(G)$ (and of course all three are explicitly presented). It is interesting to note that, in the remaining cases, one has at least

$$
\mathcal{C}^{*}(G) / \sqrt{(0)}=\mathfrak{C h}^{*}(G) / \sqrt{(0)}=\tilde{\mathcal{O}} H^{*}(G) / \sqrt{(0)}
$$

where $\sqrt{(0)}$ denotes the ideal of elements which square to 0 (not the radical). This is slightly stronger than what the general theory predicts, which is that $\mathcal{C}^{*}(G) \rightarrow \tilde{\mathcal{O}} H^{*}(G)$ is an $F$-isomorphism (in other words, it is known that for any $x \in \tilde{\mathcal{O}} H^{*}(G)$, one has $x^{2^{n}} \in \mathcal{C}^{*}(G)$ for $n$ sufficiently large).

$\diamond$ A worked out example. Let us consider $G=(\mathbb{Z} / 2)^{3}$. It is easy to perform the computations by hand (see below), but this example will serve well to illustrate what the computer does.

We have $H^{*}(G)=\mathbb{F}_{2}[x, y, z]$ where $x=w_{1}\left(r_{1}\right), y=w_{1}\left(r_{2}\right)$, and $z=w_{1}\left(r_{3}\right)$ for some 1-dimensional, real representations $r_{1}, r_{2}$ and $r_{3}$. One has

$$
S q^{1}=x^{2} \frac{\partial}{\partial x}+y^{2} \frac{\partial}{\partial y}+z^{2} \frac{\partial}{\partial z} .
$$

In the notations above, we take $A=H^{*}(G)$ and $B=\mathbb{F}_{2}\left[x^{2}, y^{2}, z^{2}\right]$. Generators for $A$ as a $B$-module are $1, x, y, z, x y, x z, y z, x y z$ (here $A=\tilde{A}$ and $B=\tilde{B}$ ).

One computes $d_{2}=S q^{1} x=x^{2}=\left(x^{2}, 0,0,0,0,0,0,0\right) \in B^{8}$, then $d_{5}=$ $S q^{1} x y=x^{2} y+x y^{2}=\left(0, y^{2}, x^{2}, 0,0,0,0\right)$, and so on.

The module of syzygies for the elements $d_{1}, \ldots, d_{8}$ has 21 generators. However as an algebra, we find that

$$
\operatorname{ker} S q^{1}=B\left[a_{1}, a_{2}, a_{3}, a_{4}\right]
$$

where

$$
\begin{gathered}
a_{1}=z^{2} x+z x^{2} \quad a_{2}=y^{2} x+y x^{2} \\
a_{3}=y^{2} z+y z^{2} \quad a_{4}=y^{2} z x+y z^{2} x+y z x^{2} .
\end{gathered}
$$

The even part of ker $S q^{1}$ is not stable under the Steenrod operations, for

$$
S q^{2}\left(a_{4}\right)=y^{4} z x+y z^{4} x+y^{2} z^{2} x^{2}+y z x^{4}
$$

and applying $S q^{1}$ to the right hand side does not give 0 .

So we move to

$$
Q_{1}=x^{4} \frac{\partial}{\partial x}+y^{4} \frac{\partial}{\partial y}+z^{4} \frac{\partial}{\partial z}
$$

Now $A=\operatorname{ker} S q^{1}$ while $B$ stays the same. The generators for $A$ as a $B$-module are $1, a_{4}, a_{3}, a_{2}, a_{1}, a_{3} a_{4}, a_{2} a_{4}, a_{2} a_{3}, a_{1} a_{4}, a_{1} a_{3}, a_{1} a_{2}, a_{2} a_{3} a_{4}, a_{1} a_{3} a_{4}, a_{1} a_{2} a_{4}$, $a_{1} a_{2} a_{3}, a_{1} a_{2} a_{3} a_{4}$.

One applies $Q_{1}$ to these and then computes the module of syzygies. There are 27,730 generators for this module, and only one (!) generator for $\operatorname{ker} Q_{1} \cap \operatorname{ker} S q^{1}$ as an algebra, namely

$$
\operatorname{ker} Q_{1} \cap \operatorname{ker} S q^{1}=B\left[y^{4} a_{1}+y^{2} x^{2} a_{1}+z^{4} a_{2}+z^{2} x^{2} a_{2}\right] .
$$

The even part of this algebra is $B$, on which every $Q_{i}$ clearly vanishes (every element of $B$ being a square in $\left.H^{*}(G)\right)$. In conclusion

$$
\tilde{\mathcal{O}} H^{*}(G)=\mathbb{F}_{2}\left[x^{2}, y^{2}, z^{2}\right] \text {. }
$$


Of course $w_{1}\left(r_{i}\right)^{2}=c_{1}\left(r_{i} \otimes \mathbb{C}\right)(i=1,2,3)$, so $\tilde{\mathcal{O}} H^{*}(G)$ is generated by Chern classes and $\mathcal{C}^{*}(G)=\tilde{\mathcal{O}} H^{*}(G)=\mathfrak{C h}^{*}(G)$.

Similar results hold for $(\mathbb{Z} / 2)^{n}$ for any $n$, and the shortest proof is by induction (in good cases one can use a Künneth formula for $\tilde{\mathcal{O}} H^{*}(G)$, see [23]).

\section{Appendix: Theoretical considerations}

In this Appendix, we expose three methods that we recommend in order to compute the Stiefel-Whitney classes. They are all "theoretical" methods, in that in each case there are serious diffulties arising when one attempts to carry the method into practice. What we have described up to this point is a way to circumvent the hard work in a lot of cases.

Throughout this section, $G$ denotes a finite group.

\section{A.1. The Atiyah-Evens approach}

Historically, Atiyah was the first to ask for a purely algebraic definition of the Chern (rather than Stiefel-Whitney) classes: see [5]. The first answer was provided by Evens, based on his multiplicative "norm", see [9]. This approach was generalized by Fulton and MacPherson in [12]. We base our discussion on [17].

The basic strategy is as follows. Suppose that $G$ is a 2-group. Then any irreducible, complex representation of $G$ is induced from a 1-dimensional representation of a subgroup of $G$ (see [24]). For real representations, the corresponding statement is: any irreducible, real representation of $G$ is induced from a representation of a subgroup $K$ of $G$ which is either 1- or 2-dimensional, and in either case obtained from a homomorphism $K \rightarrow C$, where $C$ is a finite cyclic 2-group of roots of unity in $\mathbb{C}$.

Now, the cohomology of $C$ is completely understood, of course. To be precise, when $C$ is of order 2 , it has a nontrivial real representation of real dimension 1 , and its first Stiefel-Whitney class is the only nonzero class in $H^{1}\left(C, \mathbb{F}_{2}\right)$; if $C$ has order $\geq 4$, it has a real, irreducible representation $V$ of dimension 2 obtained by viewing $C$ has a group of roots of unity, and one has $w_{1}(V)=0$ while $w_{2}(V)=c_{1}(V)$ is the only nonzero class in $H^{2}\left(C, \mathbb{F}_{2}\right)$. The representation of $K$ considered above is the "restriction" of one of these, so the Stiefel-Whitney classes may be computed by pulling back the classes in $H^{*}\left(C, \mathbb{F}_{2}\right)$ to $H^{*}\left(K, \mathbb{F}_{2}\right)$.

The difficult part is to obtain a formula for the Stiefel-Whitney (or Chern) classes of an induced representation, given the corresponding classes in the cohomology of the subgroup $K$. As noted above, Evens was the first to provide such a formula, valid only for Chern classes, while Fulton and MacPherson gave a very general statement. See also Evens and D. Kahn [10], B. Kahn [15], and Kozlowski [16]. We give the Fulton-MacPherson formula in the case when $K$ has index 2 in $G$ :

$$
w(\operatorname{Ind}(r))=N(w(r))+\sum_{d=0}^{e-1}\left[(1+\mu)^{e-d}+1\right] N\left(w_{d}(r)\right)
$$

There are quite a few notations to explain. Here $r$ is a representation of $K$, and $e$ is its real dimension; $\operatorname{Ind}(r)$ is the representation of $G$ induced by $r$. The notation $w(\rho)$ stands for the total Stiefel-Whitney class of $\rho$ :

$$
w(\rho)=1+w_{1}(\rho)+w_{2}(\rho)+\cdots
$$


which is a non-homogeneous element in the cohomology of the group of which $\rho$ is a representation. Further, $N$ is the Evens norm from $K$ to $G$ : recall that this is a map $H^{*}\left(K, \mathbb{F}_{2}\right) \rightarrow H^{*}\left(G, \mathbb{F}_{2}\right)$ which is neither additive nor degreepreserving, but it is multiplicative; moreover $N$ can be computed algebraically, see [8]. Finally, $\mu$ is the class in $H^{1}\left(G, \mathbb{F}_{2}\right)$ determined by the homomorphism $G \rightarrow G / K=\mathbb{Z} / 2 \mathbb{Z}$.

There is also a formula when $K$ has index greater than 2, but it is much more complicated. It seems easier, in this case, to consider a series of subgroups $K \subset K_{1} \subset K_{2} \subset \cdots \subset K_{n}=G$, each of index 2 in the next, and to use the formula repeatedly.

$\diamond$ Pros and cons. The advantage of this method is its relative simplicity (compare below). However, in practice, there is a serious obstacle to overcome: namely, following the method may lead one to compute the cohomology of very many subgroups of $G$, together with the Evens norm in each case. When $G$ is large, it is an understatement to say that the computation is discouragingly long.

\section{A.2. The Thom construction}

We shall now describe a discrete, or combinatorial, version of the Thom construction, which also allows the computation of Stiefel-Whitney classes.

$\diamond$ The topological side. We recall the following well-known facts (see [20]). Let $X$ be any topological space, and let $E$ be a real vector bundle over $X$. Also, let $E_{0}$ denote the complement of the zero-section in $E$. Then there is a Thom isomorphism

$$
T: H^{d}\left(X, \mathbb{F}_{2}\right) \stackrel{\simeq}{\longrightarrow} H^{n+d}\left(E, E_{0} ; \mathbb{F}_{2}\right)
$$

where $n$ is the rank of $E$. When $X$ is connected, so that there is a unique nonzero element $1 \in H^{0}\left(X, \mathbb{F}_{2}\right)$, we call $T(1) \in H^{n}\left(E, E_{0} ; \mathbb{F}_{2}\right)$ the Thom class of $E$. As it turns out, the Thom isomorphism is given by cup-multiplication with $T(1)$.

Consider then the element $S q^{i} T(1)$. It corresponds, via the Thom isomorphism, to a class in $H^{i}\left(X, \mathbb{F}_{2}\right)$. This class is $w_{i}(E)$ (indeed, this is a possible definition of the Stiefel-Whitney classes).

If now $X=B G$ and $V$ is a real representation of $G$, we may consider the universal $G$-principal bundle $E G \rightarrow B G$ and form from it the vector bundle $(E G \times V) \rightarrow B G$. Call it $E$. Then $w_{i}(V)=w_{i}(E)$.

$\diamond$ A finite CW complex acted on by $G$. Let us start with a real vector space $V$ of dimension $n$ and a set of points $A=\left\{a_{1}, \ldots, a_{m}\right\}$ whose affine span is all of $V$. We let $\Delta$ denote the convex hull of $A$, a polyhedron in $V$.

A boundary plane of $\Delta$ will mean a hyperplane of $V$ which intersects the topological boundary of $\Delta$ but not its interior. It follows from the Hahn-Banach theorem that the boundary of $\Delta$ is the union of all the boundary planes.

If $P$ is a boundary plane, then $\Delta \cap P$ is the convex hull of a subset $A^{\prime} \subset A$. When the affine span of $A^{\prime}$ is the whole of $P$, we call it a supporting plane, and $\Delta \cap P$ is called a face of $\Delta$. It is not hard to show that the boundary of $\Delta$ is the union of the faces. 
Since each $\Delta \cap P$ is itself a polyhedron in $P$, one can define inductively the $k$-faces of $\Delta$ (which are the $k-1$-faces of the faces). The $n$-faces are the vertices of $\Delta$, and they form a subset of $A$. This may be a strict subset of $A$ in general (say if $a_{2}$ is the middle of the segment from $a_{1}$ to $a_{3}$ ), but if we have chosen a Euclidean metric on $V$ and chosen the $a_{i}$ 's on the unit sphere, then none of them can belong to the interior of any $k$-face, so that the set of vertices of $\Delta$ is precisely $A$.

Topologically, $\Delta$ is an $n$-cell, and its boundary is an $n-1$-sphere. It follows that the above decomposition into $k$-faces yields a decomposition of $\Delta$ as a CWcomplex, each $k$-face giving an $n-k$-cell. We let $\Delta_{*}$ denote the corresponding mod 2 cell complex. Since we need not worry about the signs here, the boundary of a $k$-face is, quite simply, the sum of its faces. Similarly, we shall write $B d(\Delta)$ for the boundary of $\Delta$, and $B d(\Delta)_{*}$ for the corresponding complex. The discussion above is meant to show explicitly how to compute the above cell complexes in finite time.

The case of interest to us is that of an irreducible real representation $V$ of the group $G$, and $A=\left\{g_{o} v, g_{1} v, \ldots, g_{n} v\right\}$ for some nonzero $v \in V$, where the elements of $G$ have been written $g_{0}, \ldots, g_{n}$. The vector span of $A$ is $V$ since $V$ is irreducible. Assuming that $V$ is nontrivial, we see that the invariant element $g_{0} v+\cdots+g_{n} v$ is 0 , so that the barycenter of $\Delta$ is the origin in $V$, and it follows that the affine span of $A$ is $V$. We may assume that there is a $G$-equivariant Euclidean metric on $V$, so that the vertices of $\Delta$ are the points $g_{i} v$.

There is an action of $G$ on $A$ and also on $\Delta$ and $\Delta_{*}$; we see the latter as a complex of $\mathbb{F}_{2}[G]$-modules. Note that there is a homeomorphism from $\Delta$ to the unit ball in $V$, carrying its boundary to the unit sphere, defined by sending each ray emanating from the origin to a corresponding ray in the same direction, with an appropriate rescaling. Since the action of $G$ on $V$ is linear, this homeomorphism is $G$-equivariant.

$\diamond$ Resolutions. We continue with the notations for $V$ and $\Delta$. If $P_{*}$ is any projective resolution of $\mathbb{F}_{2}$ as an $\mathbb{F}_{2}[G]$-module, we define $\bar{P}_{*}$ to be the cokernel of

$$
P_{*} \otimes_{\mathbb{F}_{2}} B d(\Delta)_{*} \rightarrow P_{*} \otimes_{\mathbb{F}_{2}} \Delta_{*} .
$$

Any chain homotopy between $P_{*}$ and $Q_{*}$ yields a chain homotopy between $\bar{P}_{*}$ and $\bar{Q}_{*}$. Thus we are free to pick the projective resolution that suits our needs.

For example, we may choose for $P_{*}$ the cell complex of $E G$, the universal $G$-space. Then one knows how to put a $\mathrm{CW}$ structure on $E G \times \Delta$ so that the corresponding cell complex is just $P_{*} \otimes \Delta_{*}$, and likewise for $E G \times B d(\Delta)$. We see in this fashion that

$$
\begin{aligned}
H^{*}\left(\bar{P}_{*}\right) & \simeq H^{*}\left((E G \times \Delta) / G,(E G \times B d(\Delta)) / G ; \mathbb{F}_{2}\right) \\
& =H^{*}\left(E, E_{0} ; \mathbb{F}_{2}\right) .
\end{aligned}
$$

Here $E$ is the (total space of) the vector bundle $(E G \times V) / G$ over $B G$, as above. Also, the upper star on $H^{*}\left(\bar{P}_{*}\right)$ is meant to indicate the homology of the complex $\operatorname{Hom}_{\mathbb{F}_{2}[G]}\left(\bar{P}_{*}, \mathbb{F}_{2}\right)$. Therefore we have a Thom isomorphism and in particular, we have a Thom class $T(1)$ of degree $n$ in $H^{*}\left(\bar{P}_{*}\right)$.

On the other hand, we may pick the minimal resolution as our $P_{*}$. In this way $\bar{P}_{*}$ becomes computable. 
$\diamond$ Steenrod operations. To complete the analogy with the topological approach, we need to define the elements $S q^{i} T(1)$. There is indeed an algebraic definition of the Steenrod operations, in terms of the Evens norm map: see [8]. Strictly speaking, it is only defined for projective resolutions of a module, and our $\bar{P}_{*}$ is no such thing. However, extending the operations to this case is relatively straightforward (it is no harder than to define Steenrod operations on relative cohomology groups given the definition on regular cohomology groups).

Thus we do have elements $S q^{i} T(1) \in H^{*}\left(\bar{P}_{*}\right)$, and via the Thom isomorphism they correspond to the Stiefel-Whitney classes $w_{i}(V) \in H^{*}\left(G, \mathbb{F}_{2}\right)=$ $H^{*}(P)$. Perhaps more concretely, $w_{i}(V)$ is characterized as the only element in $H^{*}(P)$ such that the (external) cup product $w_{i}(V) \cdot T(1)=S q^{i} T(1)$.

$\diamond$ Pros and cons. On the pros side: there is no reference to any other group than $G$, and the procedure works directly for any group, not necessarily a 2group. On the cons side, one needs to know $V$ rather than just its character. So we need to find matrices representing the action of each generator of $G$. Clearly, this constitutes a rather heavy task and seems to prevent en masse calculations with lots of groups and lots of representations.

\section{A.3. The finite field trick}

$\diamond$ Universal representations. The last idea which we present is to try and find finite groups $G_{n}$ for $n=1,2, \ldots$ and for each $n$ a real representation $V_{n}$ of $G_{n}$, such that any real representation of any group $G$ is the pull-back of some $V_{n}$ under some homomorphism $f: G \rightarrow G_{n}$. Essentially, we shall explain that $G_{n}=O_{n}\left(\mathbb{F}_{3}\right)$ fits the purpose (under some hypotheses which are satisfied for us). Moreover, for a given $G$ with a representation $V$, we can take $n=\operatorname{dim} V$.

Granted this, one can compute the Stiefel-Whitney classes of $V_{n}$ in the cohomology of $G_{n}$, and pull them back using $f$. However complicated the computations with $G_{n}$ may be, once they are done for all $n \leq N$ we are able to perform rapidly many computations with any $G$ whose representations are of dimension $\leq N$. Note that the maximal dimension of an irreducible representation of $G$ grows much more slowly than the size of $G$.

$\diamond$ Reducing mod 3. We shall be concerned with real representations of real type of a finite group $G$. We recall that a real representation $r$ of a finite group can be of real, complex, or quaternion type. In the complex or quaternion case, $r$ carries a complex structure, and its Stiefel-Whitney classes can be computed from the Chern classes (see §2). In order to deal with these Chern classes, one may follow the procedure below, replacing $O_{n}$ by $G L_{n}$ and Stiefel-Whitney by Chern throughout (details left to the reader). The real case is the more delicate one.

Now, an irreducible real representation $V$ is of real type if and only if its complexification is still irreducible. Alternatively, an irreducible complex representation is the complexification of such a real representation of real type if and only if it carries a $G$-equivariant symmetric bilinear form. Here is a first application. Assume from now on that $G$ is a 2 -group. Then any irreducible complex representation is induced from a 1-dimensional representation of a subgroup. It follows that an irreducible real representation $V$ of real type is induced from a real, 1-dimensional representation of a subgroup. As a result, we see that $V$ 
may be realized with matrices with integer coefficients (indeed, involving only $1,-1$ and 0 ), and in such a way that $G$ preserves the symmetric bilinear form given by the identity matrix.

Therefore it makes sense to reduce all those entries mod 3, say (any odd prime would do). We obtain a representation $\bar{V}$ of $G$ over $\mathbb{F}_{3}$, for which the standard quadratic form is $G$-invariant. Hence we end up with a homomorphism $f: G \rightarrow O_{n}\left(\mathbb{F}_{3}\right)$.

The group $O_{n}\left(\mathbb{F}_{3}\right)$ has a canonical (defining) representation $\bar{V}_{n}$ over $\mathbb{F}_{3}$. It is tautological that, if $V$ is as above, then $\bar{V}=f^{*}\left(\bar{V}_{n}\right)$ (here $f^{*}$ means the pull-back along $f$ ).

$\diamond$ Going back to characteristic 0 . Now we use a Brauer lift of $\bar{V}_{n}$ : this is a virtual representation $V_{n}$ of $O_{n}\left(\mathbb{F}_{3}\right)$ over $\mathbb{Z}_{3}$ whose mod 3 reduction is the given $\bar{V}_{n}$. Brauer lifts always exist according to [24]. Moreover in our case Quillen in [21] has observed that $V_{n}$, when viewed as a representation over $\mathbb{C}$, carries an $O_{n}\left(\mathbb{F}_{3}\right)$-invariant quadratic form. Thus it is the complexification of a real representation and it makes sense to speak of its Stiefel-Whitney classes $w_{i}\left(V_{n}\right) \in H^{*}\left(O_{n}\left(\mathbb{F}_{3}\right), \mathbb{F}_{2}\right)$.

If one considers the virtual representation $f^{*}\left(V_{n}\right)$ of $G$, one observes that its reduction mod 3 is $f^{*}\left(\bar{V}_{n}\right)=\bar{V}$. However, since $G$ is a 2-group, the process of reducing mod 3 is an isomorphism

$$
R_{\mathbb{Q}_{3}}(G) \stackrel{\simeq}{\longrightarrow} R_{\mathbb{F}_{3}}(G)
$$

by [24]. It follows that $V$ and $f^{*}\left(V_{n}\right)$ are isomorphic over $\mathbb{Q}_{3}$; hence they are isomorphic over $\mathbb{C}$ as well; since they are each, over the complex numbers, the complexification of a (possibly virtual) real representation, it follows that the corresponding real representations are isomorphic, and have thus the same Stiefel-Whitney classes. The bottom line being that $w_{i}(V)=f^{*}\left(w_{i}\left(V_{n}\right)\right)$.

$\diamond$ Pros and cons. Computing the Stiefel-Whitney classes of sufficiently many $V_{n}$ 's is not such a tall order. First, the computation for a given $N$ yields in fact the result for all $n \leq N$ by restriction. Second, one need not take $N$ very large: say for groups of order dividing 64 , the dimension of a real representation of real type cannot be more than 4 , so dealing with $O_{4}\left(\mathbb{F}_{3}\right)$ should be enough to treat these 340 groups (to be honest, we must recall that one must also take care of $G L_{4}(k)$ for some finite field $k$ containing $\mathbb{F}_{3}$ in order to deal with the Chern classes). Finally, we note that the mod 2 cohomology of $O_{n}\left(\mathbb{F}_{3}\right)$ is rather tractable, since it is detected on a product of dihedral groups (which are well-understood), see [11]. It is an issue in practical terms, however, that we need to find explicit cocycles for these Stiefel-Whitney classes.

A significant disadvantage is, as above, that we need to know $V$ in terms of matrices, rather than just its character, if we are to compute the map $f$.

\section{References}

[1] http://www-irma.u-strasbg.fr/ guillot/research/cohomology_of_groups/index.html.

[2] http://www.math.uga.edu/ lvalero/cohointro.html.

[3] http://www.math.uni-wuppertal.de/ green/coho_v2/. 
[4] W. W. Adams and P. Loustaunau, An introduction to Gröbner bases, vol. 3 of Graduate Studies in Mathematics, American Mathematical Society, Providence, RI, 1994.

[5] M. F. AтiYah, Characters and cohomology of finite groups, Inst. Hautes Études Sci. Publ. Math., (1961), pp. 23-64.

[6] P. Brosnan, Steenrod operations in Chow theory, Trans. Amer. Math. Soc., 355 (2003), pp. 1869-1903 (electronic).

[7] J. F. Carlson, Calculating group cohomology: tests for completion, J. Symbolic Comput., 31 (2001), pp. 229-242. Computational algebra and number theory (Milwaukee, WI, 1996).

[8] J. F. Carlson, L. Townsley, L. Valeri-Elizondo, and M. Zhang, Cohomology rings of finite groups, vol. 3 of Algebras and Applications, Kluwer Academic Publishers, Dordrecht, 2003. With an appendix: Calculations of cohomology rings of groups of order dividing 64 by Carlson, Valeri-Elizondo and Zhang.

[9] L. Evens, On the Chern classes of representations of finite groups, Trans. Amer. Math. Soc., 115 (1965), pp. 180-193.

[10] L. Evens And D. S. Kahn, Chern classes of certain representations of symmetric groups, Trans. Amer. Math. Soc., 245 (1978), pp. 309-330.

[11] Z. Fiedorowicz AND S. PRIDdy, Homology of classical groups over finite fields and their associated infinite loop spaces, vol. 674 of Lecture Notes in Mathematics, Springer, Berlin, 1978.

[12] W. Fulton and R. MacPherson, Characteristic classes of direct image bundles for covering maps, Ann. of Math. (2), 125 (1987), pp. 1-92.

[13] P. Guillot, The Chow rings of $G_{2}$ and $\operatorname{Spin}(7)$, J. Reine Angew. Math., 604 (2007), pp. 137-158.

[14] — Addendum to the paper: "The Chow rings of $G_{2}$ and $\operatorname{Spin}(7)$ " [J. Reine Angew. Math. 604 (2007), 137-158;], J. Reine Angew. Math., 619 (2008), pp. 233-235.

[15] B. Kahn, Classes de Stiefel-Whitney de formes quadratiques et de représentations galoisiennes réelles, Invent. Math., 78 (1984), pp. 223-256.

[16] A. Kozlowski, The Evens-Kahn formula for the total Stiefel-Whitney class, Proc. Amer. Math. Soc., 91 (1984), pp. 309-313.

[17] — Transfers in the group of multiplicative units of the classical cohomology ring and Stiefel-Whitney classes, Publ. Res. Inst. Math. Sci., 25 (1989), pp. 59-74.

[18] J. Lannes, Sur les espaces fonctionnels dont la source est le classifiant d'un p-groupe abélien élémentaire, Inst. Hautes Études Sci. Publ. Math., (1992), pp. 135-244. With an appendix by Michel Zisman. 
[19] J. Milnor, The Steenrod algebra and its dual, Ann. of Math. (2), 67 (1958), pp. $150-171$.

[20] J. W. Milnor and J. D. Stasheff, Characteristic classes, Princeton University Press, Princeton, N. J., 1974. Annals of Mathematics Studies, No. 76 .

[21] D. Quillen, The Adams conjecture, Topology, 10 (1971), pp. 67-80.

[22] — The mod 2 cohomology rings of extra-special 2-groups and the spinor groups, Math. Ann., 194 (1971), pp. 197-212.

[23] L. Schwartz, Unstable modules over the Steenrod algebra and Sullivan's fixed point set conjecture, Chicago Lectures in Mathematics, University of Chicago Press, Chicago, IL, 1994.

[24] J.-P. Serre, Représentations linéaires des groupes finis, Hermann, Paris, revised ed., 1978.

[25] C. B. Thomas, Characteristic classes and the cohomology of finite groups, vol. 9 of Cambridge Studies in Advanced Mathematics, Cambridge University Press, Cambridge, 1986.

[26] B. Totaro, The Chow ring of a classifying space, in Algebraic $K$-theory (Seattle, WA, 1997), vol. 67 of Proc. Sympos. Pure Math., Amer. Math. Soc., Providence, RI, 1999, pp. 249-281. 